Publ. Mat. 64 (2020), 621-652

DOI: 10.5565 /PUBLMAT6422010

\title{
A SYMMETRY RESULT FOR COOPERATIVE ELLIPTIC SYSTEMS WITH SINGULARITIES
}

\author{
Stefano Biagi, Enrico Valdinoci, and Eugenio Vecchi
}

\begin{abstract}
We obtain symmetry results for solutions of an elliptic system of equation possessing a cooperative structure. The domain in which the problem is set may possess "holes" or "small vacancies" (measured in terms of capacity) along which the solution may diverge.

The method of proof relies on the moving plane technique, which needs to be suitably adapted here to take care of the complications arising from the vacancies in the domain and the analytic structure of the elliptic system.
\end{abstract}

2010 Mathematics Subject Classification: 35J47, 35B06, 31B30, 35J40.

Key words: elliptic systems, moving plane method, symmetry of solutions.

\section{Introduction and main results}

The moving plane method was introduced in the pioneer works of Aleksandrov $[\mathbf{1}, \mathbf{2}]$ in order to characterize spheres as the only closed, smooth, and connected surfaces having constant mean curvature. Afterwards, starting from the seminal paper of Serrin [29] concerning the overdetermined torsion problem, Gidas, Ni, and Nirenberg $([\mathbf{2 2}])$ and Berestycki and Nirenberg ([4]) developed further this technique in order to establish some qualitative properties of solutions of elliptic partial differential equations such as symmetry and monotonicity. The method of proof is very elegant, it relies on a beautiful geometric intuition, and its essential ingredient is the appropriate use of the maximum principle in comparing the values of the solution of the equation at two different points after a suitable reflection, which is determined by a hyperplane which gets moved up to a critical position.

In this paper we exploit the moving plane technique in order to obtain symmetry results in a setting which is not usually comprised by the classical method, since two difficulties will be taken into account. First of all, we will consider the case of general cooperative elliptic systems rather than that of a single equation, for which the moving plane

The authors are members of INdAM/GNAMPA. The second author is supported by the Australian Research Council Discovery Project 170104880 NEW "Nonlocal Equations at Work". 
technique has been settled by Troy [32]. This setting is also motivated by equations driven by polyharmonic operators with Navier boundary conditions (which, up to repeated substitutions, can be framed into elliptic systems of second order equations). Moreover, we take into account the case in which the domain presents "holes", or "cuts", or more general vacancies, along which the solution can become singular. This is an extension of our previous work [5] where we were dealing only with singularities made out of a single point, as studied in $[\mathbf{6}, \mathbf{3 1}]$ for the case of a single scalar equation.

Of course, one cannot expect a general treatment of these two situations without additional assumptions. Indeed, general elliptic systems do not satisfy the maximum principle and there is no natural order in the vectorial case, making the classical regularity theories fail in such a situation. Moreover, if the vacancies in the domain are too large, they can affect the geometry involved in the reflections and produce singularities that cannot be treated analytically in any convenient way.

To overcome these difficulties, inspired by the recent works $[\mathbf{1 8}, \mathbf{2 8}]$, we will restrict ourselves to the case of cooperative systems, in which an appropriate use of the maximum principle is possible, and consider domain vacancies that are "sufficiently small" in terms of capacities.

The precise mathematical formulation in which we work is the following. Let $m \geq 2$ be a fixed natural number. Throughout the present paper we shall be concerned with second-order cooperative (elliptic) systems of the following form

$$
\begin{cases}-\Delta u_{i}=f_{i}\left(u_{1}, \ldots, u_{m}\right) & \text { in } \Omega \backslash \Gamma, \\ u_{i}>0 & \text { in } \Omega \backslash \Gamma, \\ u_{i} \equiv 0 & \text { on } \partial \Omega,\end{cases}
$$

where $\Omega, \Gamma$, and $f_{1}, \ldots, f_{m}$ satisfy assumptions (H.1)-(H.3) below:

(H.1) $\Omega \subseteq \mathbb{R}^{n}$ is a convex open set of class $C^{\infty}$ which is bounded and symmetric with respect to the hyperplane $\Pi:=\left\{x_{1}=0\right\}$.

(H.2) $\Gamma \subseteq \Omega \cap \Pi$ is a closed set consisting of a point, if $n=2$, or verifying

$$
\operatorname{Cap}_{2}(\Gamma)=0, \quad \text { if } n \geq 3 .
$$

(H.3) $f_{1}, \ldots, f_{m} \in \operatorname{Lip}\left(\mathbb{R}^{m}\right)$ and, for every $i, j \in\{1, \ldots, m\}$ with $i \neq j$, the map

$$
\mathbb{R} \ni t_{j} \mapsto f_{i}\left(t_{1}, \ldots, t_{j-1}, t_{j}, t_{j+1}, \ldots, t_{m}\right)
$$

is non-decreasing on $(0, \infty)$ for every choice of $t_{1}, \ldots, t_{j-1}, t_{j+1}, \ldots$, $t_{n}>0$. 
We refer to Definition 2.2 for the rigorous definition of solution used in this paper. See also Definition 2.1 for the precise meaning of capacity of a set and a detailed explanation of the assumption (H.2). We want to point out that the capacitary assumption (H.2) cannot be removed nor replaced with the request that $\mathcal{L}^{n}(\Gamma)=0$; see Remark 2.6.

We are ready to state the main result of this paper.

Theorem 1.1. Let $\Omega \subseteq \mathbb{R}^{n}$ and $\Gamma \subseteq \Omega$ fulfill, respectively, assumptions (H.1) and (H.2). Moreover, let $f_{1}, \ldots, f_{m}$ satisfy assumption (H.3) and let

$$
U=\left(u_{1}, \ldots, u_{m}\right) \in H_{\mathrm{loc}}^{1}\left(\Omega \backslash \Gamma ; \mathbb{R}^{m}\right) \cap C\left(\bar{\Omega} \backslash \Gamma ; \mathbb{R}^{m}\right)
$$

be a (vector-valued) solution of the elliptic system (1.1).

Then $u_{1}, \ldots, u_{m}$ are symmetric with respect to the hyperplane $\Pi$ and increasing in the $x_{1}$-direction in $\Omega \cap\left\{x_{1}<0\right\}$. Furthermore, for every $i \in\{1, \ldots, m\}$ one has

$$
\frac{\partial u_{i}}{\partial x_{1}}(x)>0 \quad \text { for every } x \in \Omega \cap\left\{x_{1}<0\right\} .
$$

The proof of Theorem 1.1 is pretty much inspired by $[\mathbf{1 8}, \mathbf{2 8}]$. The main idea in there relies in proving the symmetry (and monotonicity) of the solution through a clever use of integral estimates. To be more precise, given the function $u$ and its reflection across a given hyperplane, one considers the positive part of their difference and shows that its gradient is actually 0 . Passing to elliptic systems this technique becomes more involved because the presence of more equations naturally leads to interactions between the solutions which have to be carefully treated. Indeed, these interactions between the different components of the (vectorial) solution cause an important loss of information on the single equations. To overcome this difficulty we will implement a sort of bootstrap procedure in which an estimate on a single component is reflected into the next one, thus producing an iterative procedure that eventually leads to a closed formula valid for all the components of the solution. We also want to stress that our result extends our previous result in [5] and it is general enough to cover a bunch of polyharmonic semilinear problems with Navier boundary conditions, even allowing for possibly singular terms.

The literature concerning symmetry results for elliptic PDEs is pretty wide and this makes it hard for us to present here an exhaustive list of references. We already mentioned the seminal papers $[\mathbf{4}, \mathbf{2 2}, \mathbf{2 9}]$ for the 
introduction and the use of the moving planes method in the elliptic PDEs setting. More recently, there has been an increasing interest in the study of elliptic PDEs (in bounded domains $\Omega \subset \mathbb{R}^{n}$ ) allowing for possible singularities, namely PDEs of the form

$$
\begin{cases}-\Delta u=\frac{1}{u^{\gamma}}+g(u) & \text { in } \Omega, \\ u=0 & \text { on } \partial \Omega,\end{cases}
$$

with $\gamma>0$. In this perspective, we want to mention [14], which is one of the first contributions dealing with singular nonlinearities, and then the more recent series of papers $[\mathbf{7}]-[\mathbf{1 0}]$. Regarding the analysis of PDEs with singularities we also highlight the seminal paper [11], where the authors carry out a qualitative study of the local behavior of a positive smooth solution near the singular set of the scalar curvature equation.

To the best of our knowledge, one of the first papers dealing with symmetry of positive solutions of elliptic PDEs in domains with holes given by a single point dates back to $[\mathbf{3 1}]$, which was then extended to slightly more general operators and sets in $[\mathbf{6}]$. The same kind of result, but with a necessary and delicate modification of the technique involved, can be also obtained in the presence of a bigger hole. In this direction, we refer to $[\mathbf{1 8}, \mathbf{2 8}]$ where the authors allow (respectively) for a hole given by a $n-2$-dimensional smooth manifold and a set of null capacity. Their ideas have also been successfully applied in the non-local setting; see $[25]$.

Let us now spend a few words concerning the case of (cooperative) elliptic systems, which can also include the case of higher order polyharmonic PDEs with Navier boundary conditions. The first result aiming at extending the results in [22] to the vectorial case is contained in [32]. Subsequently, there has been an impressive amount of contributions dealing with the validity of maximum principles (see e.g. $[\mathbf{1 6}, \mathbf{3 0}]$ ). It is also worth mentioning the papers $[\mathbf{3}, \mathbf{5}, \mathbf{1 2}, \mathbf{1 3}, \mathbf{1 5}, \mathbf{2 1}]$ (for symmetry results for semilinear polyharmonic problems and cooperative elliptic systems with or without singularities) and the very recent work [19] (where an analog of Theorem 1.1 is proved in the case of scalar quasilinear problems).

After the submission of the present manuscript we became aware of the fact that F. Esposito was about to complete the paper [17], which contains a result closely related to ours.

Plan of the paper. The present paper is organized as follows. In Section 2 we fix the notation used throughout the paper and we recall and prove a few technical results needed for the proof of Theorem 1.1, which is the content of the final Section 3. 


\section{Notations and auxiliary results}

The aim of this section is to introduce the relevant notations we shall need in the sequel and to state some auxiliary results on which we shall base the proof of Theorem 1.1. To begin with, we briefly review the precise meaning of assumption (H.2) (in the meaningful case $n \geq 3$ ).

Definition 2.1. If $U \subseteq \mathbb{R}^{n}$ is open and $E \subseteq U$ is compact, the 2-capacity of the condenser $(E, U)$ is defined as

$$
\operatorname{Cap}_{2}(E, U):=\inf \left\{\int_{U}\|\nabla u\|^{2} \mathrm{~d} x: u \in C_{0}^{\infty}(U) \text { and } u \geq 1 \text { on } E\right\} .
$$

We then say that $E$ has vanishing 2-capacity (and we write $\operatorname{Cap}_{2}(E)=0$ ) if

$$
\operatorname{Cap}_{2}(E \cap U, U)=0 \text { for every open set } U \subseteq \mathbb{R}^{n} .
$$

We recall that it can be easily proved that a compact set $E \subseteq \mathbb{R}^{n}$ has vanishing 2-capacity if and only if there exists a bounded open neighborhood $U_{0}$ of $E$ such that

$$
\mathrm{Cap}_{2}\left(E \cap U_{0}, U_{0}\right)=0 .
$$

For a proof of this fact we refer, e.g., to [24, Lemma 2.9].

We now specify what we mean by a solution of the system in (1.1).

Definition 2.2. Under the above assumptions (H.1)-(H.3), we say that a vector-valued function $U=\left(u_{1}, \ldots, u_{m}\right): \Omega \rightarrow \mathbb{R}^{m}$ is a solution of the system in (1.1) if

(1) $U \in H_{\text {loc }}^{1}\left(\Omega \backslash \Gamma ; \mathbb{R}^{m}\right) \cap C\left(\bar{\Omega} \backslash \Gamma ; \mathbb{R}^{m}\right)$, that is,

$$
u_{i} \in H_{\mathrm{loc}}^{1}(\Omega \backslash \Gamma) \cap C(\bar{\Omega} \backslash \Gamma) \text { for every } i=1, \ldots, m ;
$$

(2) for every $i \in\{1, \ldots, m\}$ one has

$$
\begin{aligned}
& \int_{\Omega}\left\langle\nabla u_{i}, \nabla \varphi\right\rangle \mathrm{d} x=\int_{\Omega} f_{i}\left(u_{1}, \ldots, u_{m}\right) \varphi \mathrm{d} x \\
& \quad \text { for every } \varphi \in C_{0}^{\infty}(\Omega \backslash \Gamma ; \mathbb{R}) ;
\end{aligned}
$$

(3) for every $i \in\{1, \ldots, m\}$ one has $u_{i}>0$ a.e. on $\Omega$ and $u_{i} \equiv 0$ on $\partial \Omega$. In this paper, if $U \subseteq \mathbb{R}^{n}$ is an arbitrary open set, the space $H_{0}^{1}(U)$ is intended as the closure of $C_{0}^{\infty}(U, \mathbb{R})$ (or, equivalently, of $\operatorname{Lip}(U) \cap$ $\left.C_{0}(U, \mathbb{R})\right)$ with respect to the norm

$$
\|u\|_{H^{1}(U)}:=\|u\|_{L^{2}(U)}+\left(\int_{U}\|\nabla u\|^{2} \mathrm{~d} x\right)^{1 / 2} .
$$


Remark 2.3. We point out that, on account of assumption (H.3), the right-hand side of any equation of the system in (1.1) is locally bounded. As a consequence, if $U=\left(u_{1}, \ldots, u_{m}\right)$ is a solution of this system of PDEs, from standard elliptic regularity we infer that

$$
u_{1}, \ldots, u_{m} \in C_{\mathrm{loc}}^{1, \alpha}(\Omega \backslash \Gamma ; \mathbb{R}) \quad \text { for every } 0<\alpha<1 .
$$

We are now ready to set the standing notations needed to perform the moving plane technique. If $\Omega \subseteq \mathbb{R}^{n}$ satisfies assumption (H.1), we set

$$
\mathbf{a}_{\Omega}:=\inf _{\Omega} x_{1} .
$$

Moreover, for every fixed $\lambda \in \mathbb{R}$ we define

$$
\Sigma_{\lambda}:=\left\{x \in \Omega: x_{1}<\lambda\right\},
$$

and we denote by $R_{\lambda}$ the symmetry with respect to the hyperplane $\Pi_{\lambda}:=\left\{x_{1}=\lambda\right\}$, i.e.,

$$
R_{\lambda}: \mathbb{R}^{n} \longrightarrow \mathbb{R}^{n}, \quad R_{\lambda}(x)=x_{\lambda}:=\left(2 \lambda-x_{1}, x_{2}, \ldots, x_{n}\right) .
$$

We explicitly notice that, since $\Omega$ is open, then the same is true of $\Omega_{\lambda}:=$ $R_{\lambda}(\Omega)$. Furthermore, since $\Omega$ is convex, we clearly have that $\Sigma_{\lambda}$ is convex and $\Sigma_{\lambda} \subseteq \Omega \cap \Omega_{\lambda}$. We collect in Lemma 2.4 some topological facts we shall need in the sequel.

Lemma 2.4. The following assertions hold true:

(1) if $E \subseteq \mathbb{R}^{n}$ is a compact set with vanishing 2 -capacity and if $U \subseteq \mathbb{R}^{n}$ is a convex open set, then $U \backslash E$ is (path-) connected;

(2) for every fixed $\lambda \in\left(\mathbf{a}_{\Omega}, 0\right)$ one has

$$
\operatorname{Cap}_{2}\left(\gamma_{\lambda}\right)=0, \text { where } \gamma_{\lambda}:=\Omega \cap\left\{x_{1}=\lambda\right\} \text {. }
$$

Proof: (1) First of all we observe that, since the set $E$ has vanishing 2-capacity, for every open neighborhood $\mathcal{O}$ of $E$ one has (see Definition 2.1)

$$
\operatorname{Cap}_{2}(E, \mathcal{O})=0 .
$$

Let then $x_{0} \neq y_{0} \in U \backslash E$ be fixed and let $\mathcal{O}_{0} \subseteq \mathbb{R}^{n}$ be an open neighborhood of $E$ such that $x_{0}, y_{0} \notin \mathcal{O}_{0}$. Moreover, let $\rho>0$ be so small that

$$
y_{0} \notin B\left(x_{0}, \rho\right), \quad B\left(x_{0}, \rho\right) \subseteq U, \quad \text { and } \quad B\left(x_{0}, \rho\right) \cap \mathcal{O}_{0}=\varnothing .
$$

We claim that there exists a point $x \in B\left(x_{0}, \rho\right) \subseteq U$ such that

(2.4) the segment $\left[x, y_{0}\right]$ joining $x$ with $y_{0}$ does not intersect $E$. 
Taking this claim for granted for a moment, we are able to complete the proof of this assertion: in fact, if $x \in B\left(x_{0}, \rho\right)$ is as in (2.4), the polygonal

$$
c:=\left[x_{0}, x\right] \cup\left[x, y_{0}\right]
$$

connects $x_{0}$ with $y_{0}$ and is contained in $U \backslash E$ (this is a straightforward consequence of $(2.3),(2.4)$, and of the fact that, by assumption, $U$ is convex).

We now turn to prove the above claim. To this end, we argue by contradiction and we assume that, for every fixed $x \in B\left(x_{0}, \rho\right)$, there exists $\bar{t}=\bar{t}_{x} \in(0,1)$ such that

$$
x+\bar{t}_{x}\left(y_{0}-x\right) \in E .
$$

If $u \in C_{0}^{\infty}\left(\mathcal{O}_{0}, \mathbb{R}\right)$ is any smooth function satisfying $u \geq 1$ on $E$, by combining (2.3) with (2.5) we obtain the following estimate (note that $\left.x \notin \mathcal{O}_{0} \supset \operatorname{supp}(u)\right)$ :

$$
\begin{aligned}
1 & \leq u\left(x+\bar{t}_{x}\left(y_{0}-x\right)\right)=u\left(x+\bar{t}_{x}\left(y_{0}-x\right)\right)-u(x) \\
& =\int_{0}^{\bar{t}_{x}}\left\langle(\nabla u)\left(x+s\left(y_{0}-x\right)\right), y_{0}-x\right\rangle \mathrm{d} s \\
& \leq\left\|y_{0}-x\right\| \int_{0}^{\bar{t}_{x}}\left\|(\nabla u)\left(x+s\left(y_{0}-x\right)\right)\right\| \mathrm{d} s
\end{aligned}
$$

(by Hölder's inequality, and setting $\kappa_{0}:=\left\|y_{0}-x_{0}\right\|+\rho$ )

$$
\leq \kappa_{0}\left(\int_{0}^{\bar{t}_{x}}\left\|(\nabla u)\left(x+s\left(y_{0}-x\right)\right)\right\|^{2} \mathrm{~d} s\right)^{1 / 2}
$$

Due to the arbitrariness of $x \in B\left(x_{0}, \rho\right)$, we are entitled to integrate both sides of $(2.6)$ on $B\left(x_{0}, \rho\right)$ with respect to $x$ : this gives (with $\omega_{n}:=$ $|B(0,1)|)$

$$
\begin{aligned}
\omega_{n} \rho^{n} & \leq \kappa_{0}^{2} \int_{B\left(x_{0}, \rho\right)}\left(\int_{0}^{\bar{t}_{x}}\left\|(\nabla u)\left(x+s\left(y_{0}-x\right)\right)\right\|^{2} \mathrm{~d} s\right) \mathrm{d} x \\
& \leq \kappa_{0}^{2} \int_{\mathbb{R}^{n}}\left(\int_{0}^{1}\left\|(\nabla u)\left(x+s\left(y_{0}-x\right)\right)\right\|^{2} \mathrm{~d} s\right) \mathrm{d} x \\
& =\kappa_{0}^{2} \int_{\mathbb{R}^{n}}\|\nabla u\|^{2} \mathrm{~d} x .
\end{aligned}
$$


Since the function $u$ was arbitrary, the above estimate implies that

$$
\inf \left\{\int_{\mathbb{R}^{n}}\|\nabla u\|^{2} \mathrm{~d} x: u \in C_{0}^{\infty}\left(\mathcal{O}_{0}, \mathbb{R}\right) \text { and } u \geq 1 \text { on } E\right\} \geq \frac{\omega_{n} \rho^{n}}{\kappa_{0}^{2}},
$$

but this is in contradiction with (2.2). Thus, (2.4) holds.

(2) If $n=2$, from the convexity of $\Omega$ (and the fact that, by assumption, $\left.\lambda>\mathbf{a}_{\Omega}\right)$ it readily follows that $\gamma_{\lambda}$ consists exactly of two points. As a consequence,

$$
\operatorname{Cap}_{2}\left(\gamma_{\lambda}\right)=0
$$

If, instead, $n \geq 3$, we claim that

$$
\gamma_{\lambda} \text { is a smooth }(n-2) \text {-dimensional manifold. }
$$

Taking this claim for granted for a moment, we are able to complete the proof of the statement: indeed, on account of (2.7), it is readily seen that the Hausdorff dimension of $\gamma_{\lambda}$ is precisely $n-2$. As a consequence, we have (see, e.g., [20])

$$
\operatorname{Cap}_{2}\left(\gamma_{\lambda}\right)=0
$$

We then turn to prove (2.7). To this end, let $\xi \in \gamma_{\lambda}$ be fixed. Since $\Omega$ is an open set of class $C^{\infty}$ (see assumption (H.1)), there exist an index $i \in\{1, \ldots, n\}$, a number $\rho>0$, and a map $\theta \in C^{\infty}\left(B\left(\xi^{\prime}, \rho\right), \mathbb{R}\right)$ (where $\left.\xi^{\prime}=\left(\xi_{1}, \ldots, \xi_{i-1}, \xi_{i+1}, \ldots, \xi_{n}\right)\right)$ such that

$$
\begin{aligned}
\partial \Omega \cap\left(\left(\xi_{i}-\rho, \xi_{i}+\rho\right) \times B\left(\xi^{\prime}, \rho\right)\right) \\
\quad=\left\{x=\left(x_{i}, x^{\prime}\right) \in\left(\xi_{i}-\rho, \xi_{i}+\rho\right) \times B\left(\xi^{\prime}, \rho\right): x_{i}=\theta\left(x^{\prime}\right)\right\} .
\end{aligned}
$$

Moreover, since $\Omega$ is convex and $\lambda>\mathbf{a}_{\Omega}$, it is quite easy to see that $\theta$ is either convex or concave on $B\left(\xi^{\prime}, \rho\right)$ and that, setting $g(x)=g\left(x_{i}, x^{\prime}\right):=$ $x_{i}-\theta\left(x^{\prime}\right)$,

$$
\nabla g(\xi) \text { is not parallel to } e_{1}=(1,0, \ldots, 0) .
$$

As a consequence, if we introduce the $\mathbb{R}^{2}$-valued function

$$
\alpha(x)=\alpha\left(x_{i}, x^{\prime}\right):=\left(x_{i}-\theta\left(x^{\prime}\right), x_{1}-\lambda\right)=\left(g(x), x_{1}-\lambda\right)
$$

(with $\left.x=\left(x_{i}, x^{\prime}\right)=\left(\xi_{i}-\rho, \xi_{i}+\rho\right) \times B\left(\xi^{\prime}, \rho\right)\right)$, we clearly have that

(a) $\alpha$ is smooth on $\mathcal{U}:=\left(\xi_{i}-\rho, \xi_{i}+\rho\right) \times B\left(\xi^{\prime}, \rho\right)$;

(b) the Jacobian matrix of $\alpha$ at $\xi$ has full rank;

(c) $\gamma_{\lambda} \cap \mathcal{U}:=\{x \in \mathcal{U}: \alpha(x)=0\}$.

Gathering together all these facts, we conclude that $\gamma_{\lambda}$ is a smooth manifold of dimension $n-2$ and the proof is finally complete. 
Remark 2.5. We explicitly observe that, on account of Lemma 2.4(1), we have that

$$
\Sigma_{\lambda} \backslash R_{\lambda}(\Gamma) \text { is connected for every } \lambda \in\left(\mathbf{a}_{\Omega}, 0\right) \text {. }
$$

In fact, since $\Gamma$ fulfills (H.2), we have that $R_{\lambda}(\Gamma)$ is compact and

$$
\operatorname{Cap}_{2}\left(R_{\lambda}(\Gamma)\right)=0 \text { for every } n \geq 2 \text {. }
$$

Moreover, as $\Omega$ is convex, the same is true of $\Sigma_{\lambda}=\Omega \cap\left\{x_{1}<\lambda\right\}$.

Actually, (2.8) can be proved in a more direct (and simpler) way by observing that

$$
R_{\lambda}(\Gamma) \subseteq\left\{x_{1}=2 \lambda\right\}
$$

In fact, since $R_{\lambda}(\Gamma)$ has vanishing 2 -capacity, it is well-known that

$$
\mathcal{H}_{\operatorname{dim}}\left(R_{\lambda}(\Gamma)\right) \leq n-2,
$$

where $\mathcal{H}_{\operatorname{dim}}\left(R_{\lambda}(\Gamma)\right)$ stands for the Hausdorff dimension of $R_{\lambda}(\Gamma)$ in $\mathbb{R}^{n}$ (see, e.g., [24]). As a consequence, there necessarily exists (at least) one point

$$
\bar{x} \in \Sigma_{\lambda} \cap\left\{x_{1}=2 \lambda\right\} \text { such that } \bar{x} \notin R_{\lambda}(\Gamma) .
$$

By combining (2.9) with (2.10) it is very easy to recognize that, if $x_{0} \neq y_{0} \in \Sigma_{\lambda} \backslash R_{\lambda}(\Gamma)$ are arbitrary, the polygonal $c=\left[x_{0}, \bar{x}\right] \cup\left[\bar{x}, y_{0}\right]$ connects $x_{0}$ with $y_{0}$ and it lays in $\Sigma_{\lambda} \backslash R_{\lambda}(\Gamma)$.

Let now $\Gamma \subseteq \Omega$ satisfy assumption (H.2), and let $f_{1}, \ldots, f_{m}$ be as in assumption (H.3). If $U=\left(u_{1}, \ldots, u_{m}\right): \Omega \rightarrow \mathbb{R}^{m}$ is any solution of the elliptic system (1.1) (according to Definition 2.2), we then introduce the following functions (defined on $\Omega_{\lambda} \backslash R_{\lambda}(\Gamma)$ ):

$$
u_{i}^{(\lambda)}:=u_{i} \circ R_{\lambda} \quad \text { and } \quad U_{\lambda}:=\left(u_{1}^{(\lambda)}, \ldots, u_{m}^{(\lambda)}\right)=U \circ R_{\lambda} .
$$

On account of Remark 2.3, we clearly have (for every $0<\alpha<1$ )

$$
U_{\lambda} \in C^{1, \alpha}\left(\Omega_{\lambda} \backslash R_{\lambda}(\Gamma) ; \mathbb{R}^{m}\right) \cap C\left(\overline{\Omega_{\lambda}} \backslash R_{\lambda}(\Gamma) ; \mathbb{R}^{m}\right) .
$$

Furthermore, since $U$ solves (1.1), we have

$$
\begin{cases}-\Delta u_{i}^{(\lambda)}=f_{i}\left(u_{1}^{(\lambda)}, \ldots, u_{n}^{(\lambda)}\right) & \text { in } \Omega_{\lambda} \backslash R_{\lambda}(\Gamma), \\ u_{i}^{(\lambda)}>0 & \text { in } \Omega_{\lambda} \backslash R_{\lambda}(\Gamma), \\ u_{i}^{(\lambda)} \equiv 0 & \text { on } \partial \Omega_{\lambda} .\end{cases}
$$


We explicitly notice that, since $U_{\lambda}$ is not of class $C^{2}$, by saying that $u_{1}^{(\lambda)}, \ldots, u_{n}^{(\lambda)}$ solve the (system of) PDEs in (2.13) we mean precisely that

$$
\int_{\Omega_{\lambda}}\left\langle\nabla u_{i}^{(\lambda)}, \nabla \varphi\right\rangle \mathrm{d} x=\int_{\Omega_{\lambda}} f_{i}\left(u_{1}^{(\lambda)}, \ldots, u_{m}^{(\lambda)}\right) \varphi \mathrm{d} x,
$$

for all $\varphi \in C_{0}^{\infty}\left(\Omega_{\lambda} \backslash R_{\lambda}(\Gamma) ; \mathbb{R}\right)$.

Remark 2.6. As already mentioned in the introduction, assumption (H.2) is somehow sharp. Let us clarify this fact with a couple of examples in the scalar case.

Example 1. In the Euclidean space $\mathbb{R}^{n}$, let $\Omega:=B(0,1)$ and let $\Gamma:=$ $\overline{B(0,1 / 2)}$. Since all the boundary points of the annulus $\mathcal{O}:=\Omega \backslash \Gamma$ are regular for the Dirichlet problem for $\Delta$, there exists a unique function $u \in$ $C^{\infty}(\mathcal{O}, \mathbb{R}) \cap C(\overline{\mathcal{O}}, \mathbb{R})$ such that

$$
\begin{cases}\Delta u=0 & \text { in } \mathcal{O}=\Omega \backslash \Gamma \\ u \equiv 0 & \text { on } \partial \Omega, \\ u(x)=e^{x_{1}} & \text { for every } x \in \partial B(0,1 / 2) .\end{cases}
$$

Owing to the classical weak and strong maximum principles, it is readily seen that $u>0$ on $\mathcal{O}=\Omega \backslash \Gamma$. Moreover, since $u$ is continuous up to $\overline{\mathcal{O}}$ and since $x \mapsto e^{x_{1}}$ is not even in $x_{1}$, we infer that $u$ cannot be symmetric with respect to the hyperplane $\Pi=\left\{x_{1}=0\right\}$.

Summing up, the function $u$ is a solution of (1.1) (with $m=1$ and $f \equiv 0$ ) which $i s$ not symmetric with respect to the hyperplane $\left\{x_{1}=0\right\}$. Notice that both $\Omega$ and $\Gamma$ are symmetric with respect to $\left\{x_{1}=0\right\}$, but $\Gamma$ has not vanishing 2-capacity (since $|\Gamma|>0$ ).

Example 2. In the Euclidean space $\mathbb{R}^{2}$, let $\Omega:=B(0,1)$ and let $\Gamma:=$ $\{0\} \times[-1 / 2,1 / 2]$. Moreover, for every fixed $n \geq 2$, we consider the (closed) rectangle

$$
R_{n}:=[-1 / n, 1 / n] \times[-1 / 2,1 / 2],
$$

and we choose a function $\varphi_{n} \in \operatorname{Lip}\left(R_{n}\right)$ such that

$$
\varphi_{n} \equiv 1 \text { on }\{1 / n\} \times[-1 / 2,1 / 2] \text { and } \varphi_{n} \equiv 2 \text { on }\{-1 / n\} \times[-1 / 2,1 / 2] \text {. }
$$

Finally, we define $\Omega_{n}:=\Omega \backslash R_{n}$. Since $\Omega_{n}$ is regular for the Dirichlet problem for $\Delta$, it is possible to find a unique function $u_{n} \in C^{\infty}\left(\Omega_{n}, \mathbb{R}\right) \cap$ $C\left(\overline{\Omega_{n}}, \mathbb{R}\right)$ such that

$$
\begin{cases}\Delta u_{n}=0 & \text { in } \Omega_{n} \\ u_{n} \equiv 0 & \text { on } \partial \Omega \\ u_{n} \equiv \varphi_{n} & \text { on } \partial R_{\epsilon}\end{cases}
$$


Furthermore, by the classical weak and strong maximum principles we have

$$
0 \leq u_{n} \leq 2 \text { on } \overline{\Omega_{n}} \quad \text { and } \quad u_{n}>0 \text { on } \Omega_{n} .
$$

We claim that the sequence $\left\{u_{n}\right\}_{n}$ has a cluster point $u_{0}$ which is a solution of (1.1) (with $m=1$ and $f \equiv 0$ ) but which is not symmetric with respect to the hyperplane $\left\{x_{1}=0\right\}$.

To prove the claim we first observe that, if $k \in \mathbb{N}$ is arbitrarily fixed and if

$$
\mathcal{O}_{k}:=\{x \in \Omega \backslash \Gamma: d(x, \partial(\Omega \backslash \Gamma))>1 / k\},
$$

there exists a natural $n_{k} \geq 2$ such that $\overline{\mathcal{O}_{k}} \subseteq \Omega_{n}$ for every $n \geq n_{k}$. As a consequence, since $\left\{u_{n}\right\}_{n \geq n_{k}}$ is a sequence of harmonic functions in $\mathcal{O}_{k}$ which is uniformly bounded on $\mathcal{O}_{k}$, there exists a harmonic function $u_{0 k}$ on $\mathcal{O}_{k}$ such that (up to a sub-sequence)

$$
\lim _{n \rightarrow \infty} u_{n}=u_{0 k} \quad \text { uniformly on every compact set of } \mathcal{O}_{k} \text {. }
$$

From this, by exploiting a suitable Cantor diagonal argument, it is then possible to find a sub-sequence $\left\{u_{n_{j}}\right\}_{j}$ of $\left\{u_{n}\right\}_{n}$ and a harmonic function $u_{0}$ on $\Omega \backslash \Gamma$ such that

$$
\lim _{j \rightarrow \infty} u_{n_{j}}=u_{0} \quad \text { uniformly on every compact set of } \Omega \backslash \Gamma \text {. }
$$

In particular, since $u_{n} \equiv 0$ on $\partial \Omega$ and $u_{n}>0$ on $\Omega_{n}$ for every $n \in \mathbb{N}$, we infer that

$$
u_{0} \equiv 0 \text { on } \partial \Omega \quad \text { and } \quad u_{0} \geq 0 \text { on } \Omega \backslash \Gamma .
$$

Let now $n \geq 2$ be arbitrarily fixed, let $P_{n}:=(-1 / n, 0)$, and let

$$
B_{n}^{-}:=B\left(P_{n}, 1 / 4\right) \cap\left\{x_{1}<-1 / n\right\} \subseteq \Omega_{n} .
$$

Since $\varphi_{n}$ is Lipschitz-continuous on $R_{n}$ and since $B_{n}^{-} \cap\left\{x_{1}=-1 / n\right\}$ is a Lipschitz portion of $\partial B_{n}^{-}$, it follows from classical results (see, e.g., Theorem 4.11 in $[\mathbf{2 3}]$ ) that

$\left|u_{n}(x)-2\right|=\left|u_{n}(x)-u_{n}\left(P_{n}\right)\right| \leq C\left|x_{1}+1 / n\right| \quad$ for any $x=\left(x_{1}, 0\right) \in B_{n}^{-}$, where $C$ is a suitable positive constant which is independent of $n$. From this, by letting $n \rightarrow \infty$ (and reminding that $u_{n_{j}} \rightarrow u_{0}$ as $j \rightarrow \infty$ pointwise on $\Omega \backslash \Gamma$ ) we get

$$
\left|u_{0}(x)-2\right| \leq C\left|x_{1}\right| \quad \text { for every } x=\left(x_{1}, 0\right) \in \Omega \backslash \Gamma \text { with } x_{1}<0 .
$$

As a consequence, we infer that

$$
\exists \lim _{\substack{x \rightarrow 0 \\ x_{1}<0}} u_{0}(x)=2 .
$$


On the other hand, if $Q_{n}:=(1 / n, 0)$ and if

$$
B_{n}^{+}:=B\left(Q_{n}, 1 / 4\right) \cap\left\{x_{1}>1 / n\right\} \subseteq \Omega_{n},
$$

by arguing exactly as before we get

$\left|u_{n}(x)-1\right|=\left|u_{n}(x)-u_{n}\left(Q_{n}\right)\right| \leq C^{\prime}\left|x_{1}-1 / n\right| \quad$ for any $x=\left(x_{1}, 0\right) \in B_{n}^{+}$,

where $C^{\prime}$ is another positive constant which is independent of $n$. From this, by letting $n \rightarrow \infty$ and by taking the limit as $x \rightarrow 0$ with $x_{1}>0$, we obtain

$$
\exists \lim _{\substack{x \rightarrow 0 \\ x_{1}>0}} u_{0}(x)=1 .
$$

Gathering together $(2.16)$ and $(2.17)$ we readily see that $u_{0}$ cannot be symmetric with respect to the hyperplane $\left\{x_{1}=0\right\}$. Moreover, since $u_{0}$ is harmonic and non-negative on $\Omega \backslash \Gamma$, by the strong maximum principle we conclude that $u_{0}>0$ on $\Omega \backslash \Gamma$.

Summing up, $u_{0}$ is a solution of (1.1) (with $m=1$ and $f \equiv 0$ ) which is not symmetric with respect to the hyperplane $\left\{x_{1}=0\right\}$. Note that, even if $|\Gamma|=0$, the set $\Gamma$ cannot have vanishing 2-capacity: in fact, its Hausdorff dimension is strictly greater than $n-2=0$.

After these preliminaries, we continue this section by constructing two sequences of functions which shall play a fundamental role in the proof of Theorem 1.1. In order to do this, we exploit some ideas contained in [18] (see, precisely, Section 2).

First of all we observe that, if $\lambda \in\left(\mathbf{a}_{\Omega}, 0\right)$ is arbitrarily fixed, on account of Lemma 2.4(2) we have $\operatorname{Cap}_{2}\left(R_{\lambda}(\Gamma)\right)=0$ (both in the case $n=$ 2 and in the case $n \geq 3$ ). As a consequence, if $\mathcal{O} \subseteq \mathbb{R}^{n}$ is any open neighborhood of $R_{\lambda}(\Gamma)$, we have

$$
\mathrm{Cap}_{2}\left(R_{\lambda}(\Gamma), \mathcal{O}\right)=0
$$

On account of (2.18), for any $k \in \mathbb{N}$ it is possible to find a function $\widetilde{\psi}_{k} \in C_{0}^{\infty}\left(\mathbb{R}^{n}, \mathbb{R}\right)$ (also depending on the fixed $\lambda$ ) such that

- $\widetilde{\psi}_{k} \geq 1$ on $R_{\lambda}(\Gamma)$ and $\operatorname{supp}\left(\widetilde{\psi}_{k}\right) \subseteq \mathcal{O}_{k}$, where

$$
\mathcal{O}_{k}=\left\{x \in \mathbb{R}^{n}: d\left(x, R_{\lambda}(\Gamma)\right)<2^{-k}\right\}
$$

- $\int_{\mathbb{R}^{n}}\left|\nabla \widetilde{\psi}_{k}\right|^{2} \mathrm{~d} x \leq 1 / k$. 
Starting from the sequence $\left\{\widetilde{\psi}_{k}\right\}_{k \in \mathbb{N}}$, we then define

$$
\psi_{k}:=T \circ \widetilde{\psi}_{k}, \quad \text { where } T(s):= \begin{cases}1 & \text { if } s<0, \\ 1-2 s & \text { if } 0 \leq s \leq 1 / 2, \\ 0 & \text { if } s>1 / 2 .\end{cases}
$$

Clearly, $\left\{\psi_{k}\right\}_{k \in \mathbb{N}} \subseteq \operatorname{Lip}\left(\mathbb{R}^{n}\right)$ and, for every fixed $k \in \mathbb{N}$, one has

$$
0 \leq \psi_{k} \leq 1
$$

$$
\begin{aligned}
& \psi_{k} \equiv 1 \text { on } \mathbb{R}^{n} \backslash \mathcal{O}_{k}, \text { and } \\
& \psi_{k} \equiv 0 \text { on a small neighborhood of } R_{\lambda}(\Gamma) .
\end{aligned}
$$

Furthermore, since $\nabla \psi_{k}=\left(T^{\prime} \circ \widetilde{\psi}_{k}\right) \cdot \nabla \widetilde{\psi}_{k}$ a.e. on $\mathbb{R}^{n}$, we also have

$$
\int_{\mathbb{R}^{n}}\left|\nabla \psi_{k}\right|^{2} \mathrm{~d} x \leq \frac{4}{k} \quad \text { for every } k \in \mathbb{N} .
$$

Arguing analogously, we construct a second sequence of functions $\left\{\phi_{h}\right\}_{h \in \mathbb{N}}$ such that, for every $h \in \mathbb{N}$, the function $\phi_{h}$ is identically 0 near the set

$$
\gamma_{\lambda}=\partial \Omega \cap \Pi_{\lambda}=\left\{x \in \partial \Omega: x_{1}=\lambda\right\} .
$$

To this end we first recall that, by Lemma 2.4(2), we have $\operatorname{Cap}_{2}\left(\gamma_{\lambda}\right)=0$. As a consequence, for every open neighborhood $\mathcal{V} \subseteq \mathbb{R}^{n}$ of $\gamma_{\lambda}$ one has

$$
\operatorname{Cap}_{2}\left(\gamma_{\lambda}, \mathcal{V}\right)=0
$$

On account of this last fact, in correspondence to every natural $h$ it is possible to construct a function $\widetilde{\phi}_{h} \in C_{0}^{\infty}\left(\mathbb{R}^{n}, \mathbb{R}\right.$ ) (also depending on the fixed $\lambda$ ) such that

- $\widetilde{\phi}_{h} \geq 1$ on $\gamma_{\lambda}$ and $\operatorname{supp}\left(\widetilde{\phi}_{h}\right) \subseteq \mathcal{V}_{h}$, where

$$
\mathcal{V}_{h}=\left\{x \in \mathbb{R}^{n}: d\left(x, \gamma_{\lambda}\right)<2^{-h}\right\}
$$

- $\int_{\mathbb{R}^{n}}\left|\nabla \widetilde{\phi}_{h}\right|^{2} \mathrm{~d} x \leq 1 / h$.

Starting from the sequence $\left\{\widetilde{\phi}_{h}\right\}_{h \in \mathbb{N}}$, we define (as above)

$$
\phi_{h}:=T \circ \widetilde{\phi}_{h}, \quad \text { where } T \text { is as in (2.19). }
$$

Clearly, $\left\{\phi_{h}\right\}_{h \in \mathbb{N}} \subseteq \operatorname{Lip}\left(\mathbb{R}^{n}\right)$ and, for every fixed $h \in \mathbb{N}$, one has

$$
0 \leq \phi_{h} \leq 1
$$

$$
\begin{aligned}
& \phi_{h} \equiv 1 \text { on } \mathbb{R}^{n} \backslash \mathcal{V}_{h}, \text { and } \\
& \phi_{h} \equiv 0 \text { on a small neighborhood of } \gamma_{\lambda} .
\end{aligned}
$$

Furthermore, since $\nabla \phi_{h}=\left(T^{\prime} \circ \widetilde{\phi}_{h}\right) \cdot \nabla \widetilde{\phi}_{h}$ a.e. on $\mathbb{R}^{n}$, we also have

$$
\int_{\mathbb{R}^{n}}\left|\nabla \phi_{h}\right|^{2} \mathrm{~d} x \leq \frac{4}{h} \quad \text { for every } h \in \mathbb{N} .
$$


Having defined the sequences $\left\{\phi_{h}\right\}_{h \in \mathbb{N}}$ and $\left\{\psi_{k}\right\}_{k \in \mathbb{N}}$, we conclude this section by stating some auxiliary results which shall be used to prove Theorem 1.1. To begin with, we state the following Lemmas 2.7 and 2.8, which are resemblant of $[\mathbf{1 8}$, Lemma 3.1].

Lemma 2.7. Let $\lambda \in\left(\mathbf{a}_{\Omega}, 0\right)$ be such that $R_{\lambda}(\Gamma) \cap \bar{\Omega}=\varnothing$ and let $\left\{\phi_{h}\right\}_{h \in \mathbb{N}}$ be the sequence defined in (2.22). Moreover, let $g \in C^{1}\left(\Sigma_{\lambda}, \mathbb{R}\right) \cap C\left(\overline{\Sigma_{\lambda}}, \mathbb{R}\right)$ be such that

$$
g \equiv 0 \text { on } \partial \Sigma_{\lambda} \cap \Pi_{\lambda} \text { and } g<0 \text { on } \partial \Sigma_{\lambda} \backslash \Pi_{\lambda} .
$$

Then the sequence of functions $\left\{\varphi_{h}\right\}_{h \in \mathbb{N}}$ defined by

$$
\varphi_{h}(x):= \begin{cases}g^{+}(x) \phi_{h}^{2}(x) & \text { if } x \in \Sigma_{\lambda}, \\ 0 & \text { if } x \in \mathbb{R}^{n} \backslash \Sigma_{\lambda},\end{cases}
$$

(here, $g^{+}=\max \{g, 0\}$ is the positive part of $g$ ) satisfies the following properties:

(1) $\left\{\varphi_{h}\right\}_{h \in \mathbb{N}} \subseteq \operatorname{Lip}\left(\mathbb{R}^{n}\right)$;

(2) $\operatorname{supp}\left(\varphi_{h}\right) \subseteq(\Omega \backslash \Gamma) \cap\left(\Omega_{\lambda} \backslash R_{\lambda}(\Gamma)\right) \cap \overline{\Sigma_{\lambda}}$ (for every fixed $\left.h \in \mathbb{N}\right)$;

(3) for every $h \in \mathbb{N}$, and a.e. on $\Omega \cup \Omega_{\lambda}$, one has

$$
\nabla \varphi_{h}=\left[\phi_{h}^{2}\left(\mathbf{1}_{\{g>0\}} \cdot \nabla g\right)+2 \phi_{h} g^{+} \cdot \nabla \phi_{h}\right] \mathbf{1}_{\operatorname{supp}\left(\varphi_{h}\right)} .
$$

In particular, $\varphi_{h} \in \operatorname{Lip}\left(\overline{\Sigma_{\lambda}}\right)$ and $\varphi_{h} \equiv 0$ on $\partial \Sigma_{\lambda}$, so that $\varphi_{h} \in H_{0}^{1}\left(\Sigma_{\lambda}\right)$.

Lemma 2.8. Let $\lambda \in\left(\mathbf{a}_{\Omega}, 0\right)$ be such that $R_{\lambda}(\Gamma) \cap \bar{\Omega} \neq \varnothing$ and let $\left\{\psi_{k}\right\}_{k \in \mathbb{N}},\left\{\phi_{h}\right\}_{h \in \mathbb{N}}$ be the sequences defined, respectively, in (2.19) and in (2.22). Moreover, let

$$
g \in C^{1}\left(\Sigma_{\lambda} \backslash R_{\lambda}(\Gamma), \mathbb{R}\right) \cap C\left(\overline{\Sigma_{\lambda}} \backslash R_{\lambda}(\Gamma), \mathbb{R}\right)
$$

be such that

$$
g \equiv 0 \text { on }\left(\partial \Sigma_{\lambda} \cap \Pi_{\lambda}\right) \backslash R_{\lambda}(\Gamma) \quad \text { and } \quad g<0 \text { on }\left(\partial \Sigma_{\lambda} \backslash \Pi_{\lambda}\right) \backslash R_{\lambda}(\Gamma) .
$$

Then the (double) sequence of functions $\left\{\varphi_{h, k}\right\}_{h, k \in \mathbb{N}}$ defined by

$$
\varphi_{h, k}(x):= \begin{cases}g^{+}(x) \phi_{h}^{2}(x) \psi_{k}^{2}(x) & \text { if } x \in \Sigma_{\lambda}, \\ 0 & \text { if } x \in \mathbb{R}^{n} \backslash \Sigma_{\lambda},\end{cases}
$$

satisfies the following properties:

(1) $\left\{\varphi_{h, k}\right\}_{h \in \mathbb{N}} \subseteq \operatorname{Lip}\left(\mathbb{R}^{n}\right)$;

(2) $\operatorname{supp}\left(\varphi_{h, k}\right) \subseteq(\Omega \backslash \Gamma) \cap\left(\Omega_{\lambda} \backslash R_{\lambda}(\Gamma)\right) \cap \overline{\Sigma_{\lambda}}($ for every $h, k \in \mathbb{N})$; 
(3) for every $h, k \in \mathbb{N}$, and a.e. on $\Omega \cup \Omega_{\lambda}$, one has

$$
\begin{aligned}
\nabla \varphi_{h, k}=\left[\phi_{h}^{2} \psi_{k}^{2}\left(\mathbf{1}_{\{g>0\}} \cdot \nabla g\right)+2 g^{+}\right. & \left(\psi_{k}^{2} \phi_{h} \cdot \nabla \phi_{h}\right. \\
& \left.\left.+\phi_{h}^{2} \psi_{k} \cdot \nabla \psi_{k}\right)\right] \mathbf{1}_{\operatorname{supp}\left(\varphi_{h, k}\right)} .
\end{aligned}
$$

In particular, $\varphi_{h, k} \in \operatorname{Lip}\left(\overline{\Sigma_{\lambda}}\right)$ and $\varphi_{h, k} \equiv 0$ on $\partial \Sigma_{\lambda}$, so that $\varphi_{h, k} \in H_{0}^{1}\left(\Sigma_{\lambda}\right)$.

We also have the following regularity result for the solutions of (1.1), which can be proved by arguing essentially as in the proof of $[\mathbf{1 8}$, Lemma 3.2].

Lemma 2.9. Let $\lambda \in\left(\mathbf{a}_{\Omega}, 0\right)$ and $i \in\{1, \ldots, m\}$ be fixed. Then

$$
\left(u_{i}-u_{i}^{(\lambda)}\right)^{+} \in H_{0}^{1}\left(\Sigma_{\lambda}\right) .
$$

Furthermore, if $\lambda$ is such that $R_{\lambda}(\Gamma) \cap \bar{\Omega}=\varnothing$, then

$$
\lim _{h \rightarrow \infty} \varphi_{h}=\left(u_{i}-u_{i}^{(\lambda)}\right)^{+} \text {in } H_{0}^{1}\left(\Sigma_{\lambda}\right),
$$

where $\varphi_{h}$ is as in Lemma 2.7, with $g=u_{i}-u_{i}^{(\lambda)}$. If, instead, $\lambda$ is such that $R_{\lambda}(\Gamma) \cap \bar{\Omega} \neq \varnothing$, then

$$
\lim _{h, k \rightarrow \infty} \varphi_{h, k}=\left(u_{i}-u_{i}^{(\lambda)}\right)^{+} \text {in } H_{0}^{1}\left(\Sigma_{\lambda}\right),
$$

where $\varphi_{h, k}$ is as in Lemma 2.8, with $g=u_{i}-u_{i}^{(\lambda)}$.

Finally, we prove a technical lemma which will be used in the proof of Theorem 1.1.

Lemma 2.10. Let $n \geq 2$ and let $U \subseteq \mathbb{R}^{n}$ be an open and bounded set with Lipschitz boundary. There exists a real constant $\Theta=\Theta_{n}>0$, independent of $U$, such that

$$
\|u\|_{L^{2}(U)} \leq \Theta|U|^{1 / n}\|\nabla u\|_{L^{2}(U)} \quad \text { for every } u \in H_{0}^{1}(U) .
$$

Proof: We first prove (2.30) for a function $v \in C_{0}^{\infty}(U)$ (not identically vanishing on $U$ ). Since, in particular, we can think of $v$ as a function belonging to $C_{0}^{\infty}\left(\mathbb{R}^{n}\right)$, by applying the Nash inequality (see, e.g., [26]) and Hölder's inequality we get

$$
\begin{aligned}
\|v\|_{L^{2}(U)}^{1+2 / n} & =\|v\|_{L^{2}\left(\mathbb{R}^{n}\right)}^{1+2 / n} \leq \Theta\|v\|_{L^{1}\left(\mathbb{R}^{n}\right)}\|\nabla v\|_{L^{2}\left(\mathbb{R}^{n}\right)} \\
& \leq \Theta|U|^{1 / n}\|v\|_{L^{2}(U)}^{2 / n}\|\nabla v\|_{L^{2}(U)},
\end{aligned}
$$

where $\Theta>0$ is a real constant only depending on the dimension $n$. As a consequence, since we have assumed that $v \not \equiv 0$ on $U$, we obtain

$$
\|v\|_{L^{2}(U)} \leq \Theta|U|^{1 / n}\|\nabla v\|_{L^{2}(U)} .
$$


The proof of (2.30) for a general $u \in H_{0}^{1}(U)$ follows by a density argument.

\section{Proof of Theorem 1.1}

In the present section we give the proof of our Theorem 1.1. In doing this, we retain all the notations introduced in the preceding sections.

Proof (of Theorem 1.1): For every $\lambda \in\left(\mathbf{a}_{\Omega}, 0\right)$, we consider the functions

$$
w_{i}^{(\lambda)}:=u_{i}-u_{i}^{(\lambda)}, \quad W_{\lambda}:=\left(w_{1}^{(\lambda)}, \ldots, w_{n}^{(\lambda)}\right)=U-U_{\lambda} .
$$

Taking into account the regularity of $U$ and of $U_{\lambda}$ (see, respectively, Definition 2.2 and (2.12)), and reminding that $\Sigma_{\lambda} \subseteq \Omega \cap \Omega_{\lambda}$, it is readily seen that (for any $0<\alpha<1$ )

$$
W_{\lambda} \in C^{1, \alpha}\left(\Sigma_{\lambda} \backslash R_{\lambda}(\Gamma) ; \mathbb{R}^{m}\right) \cap C\left(\overline{\Sigma_{\lambda}} \backslash R_{\lambda}(\Gamma) ; \mathbb{R}^{m}\right) .
$$

Furthermore, since $U$ solves (1.1) and $U_{\lambda}$ solves (2.14) we have (note that, as $\Omega$ is convex, the reflection of $\partial \Sigma_{\lambda} \backslash \Pi_{\lambda}$ with respect to $\Pi_{\lambda}$ is entirely contained in $\Omega$ )

$$
\begin{cases}-\Delta w_{i}^{(\lambda)}=\sum_{j=1}^{m} c_{i j}(x ; \lambda) w_{j}^{(\lambda)} & \text { on } \Sigma_{\lambda} \backslash R_{\lambda}(\Gamma), \\ w_{i}^{(\lambda)}<0 & \text { on }\left(\partial \Sigma_{\lambda} \backslash \Pi_{\lambda}\right) \backslash R_{\lambda}(\Gamma), \\ w_{i}^{(\lambda)} \equiv 0 & \text { on } \partial \Sigma_{\lambda} \cap \Pi_{\lambda},\end{cases}
$$

where $c_{i 1}(\cdot ; \lambda), \ldots, c_{i m}(\cdot ; \lambda): \Sigma_{\lambda} \backslash R_{\lambda}(\Gamma) \rightarrow \mathbb{R}$ are defined as follows:

$$
c_{i j}(x ; \lambda):= \begin{cases}\frac{f_{i}(U(x))-f_{i}\left(U_{\lambda}(x)\right)}{u_{j}(x)-u_{j}^{(\lambda)}(x)} & \text { if } u_{i}(x) \neq u_{i}^{(\lambda)}(x), \\ 0 & \text { otherwise. }\end{cases}
$$

As for the case of $U$ and $U_{\lambda}$, since $W_{\lambda}$ is not of class $C^{2}$ on $\Sigma_{\lambda}$, by saying that $w_{1}^{(\lambda)}, \ldots, w_{m}^{(\lambda)}$ solve the system of PDEs in (3.2) we mean precisely that

$$
\begin{aligned}
& \int_{\Sigma_{\lambda}}\left\langle\nabla w_{i}^{(\lambda)}, \nabla \varphi\right\rangle \mathrm{d} x=\sum_{j=1}^{m} \int_{\Sigma_{\lambda}} c_{i j}(\cdot ; \lambda) w_{j}^{(\lambda)} \varphi \mathrm{d} x \\
& \qquad \text { for all } \varphi \in C_{0}^{\infty}\left(\Sigma_{\lambda} \backslash R_{\lambda}(\Gamma), \mathbb{R}\right) .
\end{aligned}
$$


Moreover, on account of assumption (H.3), we see that

(a) $c_{i j}(\cdot ; \lambda) \geq 0$ for every $i \in\{1, \ldots, m\}$ and every $j \neq i$;

(b) there exists a real constant $\mathbf{c}_{f}>0$ such that

(3.5) $0 \leq c_{i j}(\cdot ; \lambda) \leq \mathbf{c}_{f} \quad$ for every $i, j \in\{1, \ldots, m\}$ and every $\lambda \in\left(\mathbf{a}_{\Omega}, 0\right)$.

According to the well-established moving planes technique, we now define

$$
\begin{aligned}
\mathcal{I}:= & \left\{\lambda \in\left(\mathbf{a}_{\Omega}, 0\right): w_{i}^{(t)}<0 \text { on } \Sigma_{t} \backslash R_{t}(\Gamma)\right. \\
& \text { for all } \left.t \in\left(\mathbf{a}_{\Omega}, \lambda\right) \text { and for all } i \in\{1, \ldots, m\}\right\} \text { and } \lambda_{0}:=\sup \mathcal{I} .
\end{aligned}
$$

Our aim is to prove that $\mathcal{I} \neq \varnothing$ and that $\lambda_{0}=0$. From now on, in order to ease the readability, we split the proof into different steps.

Step I: In this step we prove that $\mathcal{I} \neq \varnothing$ and that $\lambda_{0}>\mathbf{a}_{\Omega}$. We fix $t_{0} \in\left(\mathbf{a}_{\Omega}, 0\right)$ such that $R_{t_{0}}(\Gamma) \subset \Omega^{c}$. Necessarily, we have that $R_{t}(\Gamma) \subset \Omega^{c}$ for every $t \in\left(\mathbf{a}_{\Omega}, t_{0}\right)$. Now, for every $i=1, \ldots, m$ we consider the function $\varphi_{i, h}: \Omega \rightarrow \mathbb{R}$ defined as

$$
\varphi_{i, h}:=\left(w_{i}^{(t)}\right)^{+} \phi_{h}^{2} \mathbf{1}_{\Sigma_{t}}
$$

where $\left\{\phi_{h}\right\}_{h \in \mathbb{N}}$ is as in (2.22). The next computation is analogous to that in Step II, where a more complicated test function is involved. However, we present it in all the details for the sake of completeness. By density, we can use $\varphi_{i, h}$ as a test function in (3.4), finding

$$
\begin{aligned}
& \int_{\Sigma_{t}}\left\langle\nabla w_{i}^{(t)}, \nabla\left(w_{i}^{(t)}\right)^{+}\right\rangle \phi_{h}^{2} \mathrm{~d} x \\
& \quad=-2 \int_{\Sigma_{t}}\left\langle\nabla w_{i}^{(t)}, \nabla \phi_{h}\right\rangle\left(w_{i}^{(t)}\right)^{+} \phi_{h} \mathrm{~d} x+\sum_{j=1}^{m} \int_{\Sigma_{t}} c_{i j}(\cdot ; t)\left(w_{i}^{(t)}\right)^{+} w_{j}^{(t)} \phi_{h}^{2} \mathrm{~d} x
\end{aligned}
$$

(by using Cauchy-Schwarz's inequality, (3.5), and (2.23))

$$
\leq 2 \int_{\Sigma_{t}}\left|\nabla w_{i}^{(t)}\right|\left|\nabla \phi_{h}\right|\left(w_{i}^{(t)}\right)^{+} \phi_{h} \mathrm{~d} x+\mathbf{c}_{f} \sum_{j=1}^{m} \int_{\Sigma_{t}}\left(w_{j}^{(t)}\right)^{+}\left(w_{i}^{(t)}\right)^{+} \mathrm{d} x .
$$


On the other hand, since $\nabla\left(w_{i}^{(t)}\right)^{+}=\nabla w_{i}^{(t)} \mathbf{1}_{\left\{w_{i}^{(t)}>0\right\}}$, we can write

$$
\begin{aligned}
& \int_{\Sigma_{t}}\left|\nabla\left(w_{i}^{(t)}\right)^{+}\right|^{2} \phi_{h}^{2} \mathrm{~d} x \\
& \quad \leq 2 \int_{\Sigma_{t}}\left|\nabla\left(w_{i}^{(t)}\right)^{+}\right|\left|\nabla \phi_{h}\right|\left(w_{i}^{(t)}\right)^{+} \phi_{h} \mathrm{~d} x+\mathbf{c}_{f} \sum_{j=1}^{m} \int_{\Sigma_{t}}\left(w_{j}^{(t)}\right)^{+}\left(w_{i}^{(t)}\right)^{+} \mathrm{d} x
\end{aligned}
$$

(by Young's inequality)

$$
\begin{aligned}
\leq & \frac{1}{2} \int_{\Sigma_{t}}\left|\nabla\left(w_{i}^{(t)}\right)^{+}\right|^{2} \phi_{h}^{2} \mathrm{~d} x+2 \int_{\Sigma_{t}}\left|\nabla \phi_{h}\right|^{2}\left[\left(w_{i}^{(t)}\right)^{+}\right]^{2} \mathrm{~d} x \\
& +\mathbf{c}_{f} \sum_{j=1}^{m} \int_{\Sigma_{t}}\left(w_{j}^{(t)}\right)^{+}\left(w_{i}^{(t)}\right)^{+} \mathrm{d} x .
\end{aligned}
$$

We now observe that, since $u_{1}, \ldots, u_{m}$ are non-negative in $\Omega \backslash \Gamma$, we have

$$
\left(w_{i}^{(t)}\right)^{+}=\left(u_{i}-u_{i}^{(t)}\right)^{+} \leq u_{i} \quad \text { on } \Sigma_{t} .
$$

As a consequence, since $u_{i}$ is continuous on $\overline{\Sigma_{t}} \subseteq \bar{\Omega} \backslash \Gamma$, from (2.24) we obtain

$$
\frac{1}{2} \int_{\Sigma_{t}}\left|\nabla\left(w_{i}^{(t)}\right)^{+}\right|^{2} \phi_{h}^{2} \mathrm{~d} x \leq 8\left\|u_{i}\right\|_{L^{\infty}\left(\Sigma_{t}\right)}^{2} \cdot \frac{1}{h}+\mathbf{c}_{f} \sum_{j=1}^{m} \int_{\Sigma_{t}}\left(w_{j}^{(t)}\right)^{+}\left(w_{i}^{(t)}\right)^{+} \mathrm{d} x .
$$

By Fatou's Lemma, sending $h \rightarrow \infty$ we get

$$
\frac{1}{2} \int_{\Sigma_{t}}\left|\nabla\left(w_{i}^{(t)}\right)^{+}\right|^{2} \mathrm{~d} x \leq \mathbf{c}_{f} \sum_{j=1}^{m} \int_{\Sigma_{t}}\left(w_{j}^{(t)}\right)^{+}\left(w_{i}^{(t)}\right)^{+} \mathrm{d} x .
$$

By Hölder's inequality on every term on the right hand side, we get

$$
\frac{1}{2}\left\|\nabla\left(w_{i}^{(t)}\right)^{+}\right\|_{L^{2}\left(\Sigma_{t}\right)}^{2} \mathrm{~d} x \leq \mathbf{c}_{f} \sum_{j=1}^{m}\left\|\left(w_{j}^{(t)}\right)^{+}\right\|_{L^{2}\left(\Sigma_{t}\right)}\left\|\left(w_{i}^{(t)}\right)^{+}\right\|_{L^{2}\left(\Sigma_{t}\right)} .
$$

From this, by using (2.30) (on every term on the right hand side), for every $t \in\left(\mathbf{a}_{\Omega}, t_{0}\right)$ and every index $i \in\{1, \ldots, m\}$ we get

$$
\frac{1}{2}\left\|\nabla\left(w_{i}^{(t)}\right)^{+}\right\|_{L^{2}\left(\Sigma_{t}\right)}^{2} \mathrm{~d} x \leq \mathbf{c}_{f} \theta_{n}^{2}\left(\Sigma_{t}\right) \sum_{j=1}^{m}\left\|\nabla\left(w_{j}^{(t)}\right)^{+}\right\|_{L^{2}\left(\Sigma_{t}\right)}\left\|\nabla\left(w_{i}^{(t)}\right)^{+}\right\|_{L^{2}\left(\Sigma_{t}\right)},
$$


where we have introduced the notation (repeatedly used in the sequel)

$$
\theta_{n}\left(\Sigma_{t}\right):=\Theta\left|\Sigma_{t}\right|^{1 / n} \quad(\text { with } \Theta>0 \text { is as in Lemma 2.10). }
$$

Now, if $\left\|\nabla\left(w_{i}^{(t)}\right)^{+}\right\|_{L^{2}\left(\Sigma_{t}\right)} \neq 0$, from the above inequality we immediately get

$$
\frac{1}{2}\left\|\nabla\left(w_{i}^{(t)}\right)^{+}\right\|_{L^{2}\left(\Sigma_{t}\right)} \leq \mathbf{c}_{f} \theta_{n}^{2}\left(\Sigma_{t}\right) \sum_{j=1}^{m}\left\|\nabla\left(w_{j}^{(t)}\right)^{+}\right\|_{L^{2}\left(\Sigma_{t}\right)} .
$$

On the other hand, since (3.7) is trivially satisfied when $\left\|\nabla\left(w_{i}^{(t)}\right)^{+}\right\|_{L^{2}\left(\Sigma_{t}\right)}=$ 0 , we conclude that such an inequality holds true for every $i \in\{1, \ldots, m\}$ and every $t \in\left(\mathbf{a}_{\Omega}, t_{0}\right)$.

We now aim at proving the following assertion: for every fixed $k \in$ $\{1, \ldots, m-1\}$ there exist $t_{k} \in\left(\mathbf{a}_{\Omega}, t_{0}\right)$ and a real constant $C_{k}=C_{k}\left(m, \mathbf{c}_{f}\right)>$ 0 such that

$$
\left\|\nabla\left(w_{i}^{(t)}\right)^{+}\right\|_{L^{2}\left(\Sigma_{t}\right)} \leq C_{k} \theta_{n}^{2}\left(\Sigma_{t}\right) \sum_{j \geq i+1}\left\|\nabla\left(w_{j}^{(t)}\right)^{+}\right\|_{L^{2}\left(\Sigma_{t}\right)},
$$

for all $1 \leq i \leq k$ and every $t \in\left(\mathbf{a}_{\Omega}, t_{k}\right)$. To prove (3.8) we argue by (finite) induction and we start with $k=1$. By (3.7) we have

$$
\left(\frac{1}{2}-\mathbf{c}_{f} \theta_{n}^{2}\left(\Sigma_{t}\right)\right)\left\|\nabla\left(w_{1}^{(t)}\right)^{+}\right\|_{L^{2}\left(\Sigma_{t}\right)} \leq \mathbf{c}_{f} \theta_{n}^{2}\left(\Sigma_{t}\right) \sum_{j \geq 2}\left\|\nabla\left(w_{j}^{(t)}\right)^{+}\right\|_{L^{2}\left(\Sigma_{t}\right)} .
$$

Since $\theta_{n}\left(\Sigma_{t}\right) \rightarrow 0$ as $t \rightarrow \mathbf{a}_{\Omega}$, it is possible to find $t_{1} \in\left(\mathbf{a}_{\Omega}, t_{0}\right)$ such that

$$
\frac{1}{2}-\mathbf{c}_{f} \theta_{n}^{2}\left(\Sigma_{t}\right)>\frac{1}{4} \quad \text { for every } t \in\left(\mathbf{a}_{\Omega}, t_{1}\right) .
$$

As a consequence, we obtain

$$
\left\|\nabla\left(w_{1}^{(t)}\right)^{+}\right\|_{L^{2}\left(\Sigma_{t}\right)} \leq 4 \mathbf{c}_{0} \theta_{n}^{2}\left(\Sigma_{t}\right) \sum_{j \geq 2}\left\|\nabla\left(w_{j}^{(t)}\right)^{+}\right\|_{L^{2}\left(\Sigma_{t}\right)},
$$

which is precisely (3.8) for $i=1$ (with $C_{1}=4 \mathbf{c}_{0}$ ). Let us now suppose that (3.8) holds for a certain index $k \in\{1, \ldots, m-2\}$ and, by shrinking $t_{k}$ if necessary, let us also assume that $\theta_{n}\left(\Sigma_{t}\right)<1$ for all $t \in\left(\mathbf{a}_{\Omega}, t_{k}\right)$. 
By (3.7) (with $i=k+1$ ), we then have

$$
\begin{gathered}
\left(\frac{1}{2}-\mathbf{c}_{f} \theta_{n}^{2}\left(\Sigma_{t}\right)\right)\left\|\nabla\left(w_{k+1}^{(t)}\right)^{+}\right\|_{L^{2}\left(\Sigma_{t}\right)} \leq \mathbf{c}_{f} \theta_{n}^{2}\left(\Sigma_{t}\right) \sum_{i \neq k+1}\left\|\nabla\left(w_{i}^{(t)}\right)^{+}\right\|_{L^{2}\left(\Sigma_{t}\right)} \\
=\mathbf{c}_{f} \theta_{n}^{2}\left(\Sigma_{t}\right)\left(\sum_{i=1}^{k}\left\|\nabla\left(w_{i}^{(t)}\right)^{+}\right\|_{L^{2}\left(\Sigma_{t}\right)}+\sum_{i \geq k+2}\left\|\nabla\left(w_{i}^{(t)}\right)^{+}\right\|_{L^{2}\left(\Sigma_{t}\right)}\right)
\end{gathered}
$$

(by (3.8), which we are assuming to hold for the index $k$ )

$$
\begin{gathered}
\leq \mathbf{c}_{f} \theta_{n}^{2}\left(\Sigma_{t}\right)\left(C_{k} \sum_{i=1}^{k} \sum_{j \geq i+1}\left\|\nabla\left(w_{j}^{(t)}\right)^{+}\right\|_{L^{2}\left(\Sigma_{t}\right)}+\sum_{i \geq k+2}\left\|\nabla\left(w_{i}^{(t)}\right)^{+}\right\|_{L^{2}\left(\Sigma_{t}\right)}\right) \\
\leq \mathbf{c}_{f} \theta_{n}^{2}\left(\Sigma_{t}\right)\left(m C_{k} \sum_{j=2}^{k+1}\left\|\nabla\left(w_{j}^{(t)}\right)^{+}\right\|_{L^{2}\left(\Sigma_{t}\right)}\right. \\
\left.+\left(k C_{k}+1\right) \sum_{i \geq k+2}\left\|\nabla\left(w_{i}^{(t)}\right)^{+}\right\|_{L^{2}\left(\Sigma_{t}\right)}\right)=:(\star) .
\end{gathered}
$$

We now perform a backward induction argument to show that, as a consequence of the validity of (3.8) for the index $k$, the following fact holds: for every fixed $j \in\{1, \ldots, k\}$, it is possible to find a real constant $\mathcal{C}_{j}=\mathcal{C}_{j}\left(m, k, \mathbf{c}_{f}\right)>0$ such that

$$
\left\|\nabla\left(w_{j}^{(t)}\right)^{+}\right\|_{L^{2}\left(\Sigma_{t}\right)} \leq \mathcal{C}_{j} \theta_{n}^{2}\left(\Sigma_{t}\right) \sum_{r \geq k+1}\left\|\nabla\left(w_{r}^{(t)}\right)^{+}\right\|_{L^{2}\left(\Sigma_{t}\right)}
$$

for every $t \in\left(\mathbf{a}_{\Omega}, t_{k}\right)$. For $j=k$, (3.10) follows immediately from (3.8) by taking $i=k$ (with $\mathcal{C}_{k}:=C_{k}$ ). We then suppose the existence of an index $j \in\{2, \ldots, k\}$ such that (3.10) holds for every $j \leq r \leq k$, and we exploit once again (3.8) (with $i=j-1 \leq k-1$ ):

$$
\begin{aligned}
& \left\|\nabla\left(w_{j-1}^{(t)}\right)^{+}\right\|_{L^{2}\left(\Sigma_{t}\right)} \leq C_{k} \theta_{n}^{2}\left(\Sigma_{t}\right) \sum_{r \geq j}\left\|\nabla\left(w_{r}^{(t)}\right)^{+}\right\|_{L^{2}\left(\Sigma_{t}\right)} \\
& =C_{k} \theta_{n}^{2}\left(\Sigma_{t}\right)\left(\sum_{r=j}^{k}\left\|\nabla\left(w_{r}^{(t)}\right)^{+}\right\|_{L^{2}\left(\Sigma_{t}\right)}+\sum_{r \geq k+1}\left\|\nabla\left(w_{r}^{(t)}\right)^{+}\right\|_{L^{2}\left(\Sigma_{t}\right)}\right)
\end{aligned}
$$

(since $(3.10)$ holds for $j \leq r \leq k$, and $\theta_{n}\left(\Sigma_{t}\right)<1$ )

$$
\leq C_{k}\left(m \max _{j \leq r \leq k}\left(C_{r}\right)+1\right) \theta_{n}^{2}\left(\Sigma_{t}\right) \sum_{r \geq k+1}\left\|\nabla\left(w_{r}^{(t)}\right)^{+}\right\|_{L^{2}\left(\Sigma_{t}\right)},
$$


so that (3.10) holds true also for $j-1$. By the Induction Principle, we then conclude that estimate $(3.10)$ is valid for every $j=1, \ldots, k$, as claimed.

With (3.10) at hand, we now continue the estimate (3.9): reminding that, by the choice of $t_{k}$, we have $\theta_{n}\left(\Sigma_{t}\right)<1$ for every $t \in\left(\mathbf{a}_{\Omega}, t_{k}\right)$, we have

$$
(\star) \leq M_{k} \theta_{n}^{2}\left(\Sigma_{t}\right) \sum_{j \geq k+1}\left\|\nabla\left(w_{j}^{(t)}\right)^{+}\right\|_{L^{2}\left(\Sigma_{t}\right)},
$$

where $M_{k}=M_{k}\left(m, \mathbf{c}_{f}\right)>0$ is a suitable quantity. As a consequence, we obtain

$$
\begin{aligned}
\left(\frac{1}{2}-\mathbf{c}_{f} \theta_{n}^{2}\left(\Sigma_{t}\right)-M_{k} \theta_{n}^{2}\left(\Sigma_{t}\right)\right) & \left\|\nabla\left(w_{k+1}^{(t)}\right)^{+}\right\|_{L^{2}\left(\Sigma_{t}\right)} \\
& \leq M_{k} \theta_{n}^{2}\left(\Sigma_{t}\right) \sum_{j \geq k+2}\left\|\nabla\left(w_{j}^{(t)}\right)^{+}\right\|_{L^{2}\left(\Sigma_{t}\right)} .
\end{aligned}
$$

Finally, since $\theta_{n}\left(\Sigma_{t}\right) \rightarrow 0$ as $t \rightarrow \mathbf{a}_{\Omega}$, we infer the existence of $\bar{t} \in\left(\mathbf{a}_{\Omega}, t_{0}\right)$ such that

$$
\frac{1}{2}-\mathbf{c}_{f} \theta_{n}^{2}\left(\Sigma_{t}\right)-M_{k} \theta_{n}^{2}\left(\Sigma_{t}\right)>\frac{1}{4} \quad \text { for every } t \in\left(\mathbf{a}_{\Omega}, \bar{t}\right) .
$$

From this, we obviously derive the estimate (valid for $t \in\left(\mathbf{a}_{\Omega}, \bar{t}\right)$ )

$$
\left\|\nabla\left(w_{k+1}^{(t)}\right)^{+}\right\|_{L^{2}\left(\Sigma_{t}\right)} \leq 4 M_{k} \theta_{n}^{2}\left(\Sigma_{t}\right) \sum_{j \geq k+2}\left\|\nabla\left(w_{j}^{(t)}\right)^{+}\right\|_{L^{2}\left(\Sigma_{t}\right)} .
$$

Taking as $t_{k+1}:=\min \left\{t_{k}, \bar{t}\right\}$, and setting $C_{k+1}:=\max \left\{C_{k}, 4 M_{k}\right\}$, we then obtain

$$
\left\|\nabla\left(w_{i}^{(t)}\right)^{+}\right\|_{L^{2}\left(\Sigma_{t}\right)} \leq C_{k+1} \theta_{n}^{2}\left(\Sigma_{t}\right) \sum_{j \geq i+1}\left\|\nabla\left(w_{i}^{(t)}\right)^{+}\right\|_{L^{2}\left(\Sigma_{t}\right)},
$$

for all $1 \leq i \leq k+1$ and every $t \in\left(\mathbf{a}_{\Omega}, t_{k+1}\right)$, so that (3.8) holds true also for $k+1$. By the Induction Principle, we conclude that estimate (3.8) is valid for every $k=1, \ldots, m-2$, as claimed.

Now we have established (3.8), we are able to complete the proof this step. In fact, since said (3.8) holds true for $k=m-1$, a (finite) backward induction argument shows the existence of a real constant $\mathcal{C}_{m}=\mathcal{C}_{m}\left(\mathbf{c}_{0}\right)>0$ such that

$$
\left\|\nabla\left(w_{j}^{(t)}\right)^{+}\right\|_{L^{2}\left(\Sigma_{t}\right)} \leq \mathcal{C}_{m} \theta_{n}^{2}\left(\Sigma_{t}\right)\left\|\nabla\left(w_{m}^{(t)}\right)^{+}\right\|_{L^{2}\left(\Sigma_{t}\right)},
$$


for all $1 \leq j \leq m-1$ and every $t \in\left(\mathbf{a}_{\Omega}, t_{m-1}\right)$. Gathering together (3.12) and (3.7) (with $i=m$ ), for any $t \in\left(\mathbf{a}_{\Omega}, t_{m-1}\right)$ we get

$$
\frac{1}{2}\left\|\nabla\left(w_{m}^{(t)}\right)^{+}\right\|_{L^{2}\left(\Sigma_{t}\right)} \leq \mathbf{c}_{f} \theta_{n}^{2}\left(\Sigma_{t}\right)\left(m \mathcal{C}_{m} \theta_{n}^{2}\left(\Sigma_{t}\right)+1\right)\left\|\nabla\left(w_{m}^{(t)}\right)^{+}\right\|_{L^{2}\left(\Sigma_{t}\right)} .
$$

Since $\theta_{n}\left(\Sigma_{t}\right) \rightarrow 0$ as $t \rightarrow \mathbf{a}_{\Omega}$, there exists $\tau_{0} \in\left(\mathbf{a}_{\Omega}, t_{m-1}\right)$ such that

$$
\mathbf{c}_{f} \theta_{n}^{2}\left(\Sigma_{t}\right)\left(m \mathcal{C}_{m} \theta_{n}^{2}\left(\Sigma_{t}\right)+1\right)<\frac{1}{4} \quad \text { for every } t \in\left(\mathbf{a}_{\Omega}, \tau_{0}\right) .
$$

As a consequence, we obtain

$$
\left\|\nabla\left(w_{m}^{(t)}\right)\right\|_{L^{2}\left(\Sigma_{t}\right)}=0 \quad \text { for every } t \in\left(\mathbf{a}_{\Omega}, \tau_{0}\right) .
$$

On account of (3.8), this proves that

$$
\left\|\nabla\left(w_{1}^{(t)}\right)\right\|_{L^{2}\left(\Sigma_{t}\right)}=\cdots=\left\|\nabla\left(w_{m}^{(t)}\right)\right\|_{L^{2}\left(\Sigma_{t}\right)}=0 \quad \text { for every } t \in\left(\mathbf{a}_{\Omega}, \tau_{0}\right) .
$$

As a consequence, by Lemma 2.10 (and since $W_{t}$ is continuous on $\Sigma_{t}$ ) we get

$$
u_{i}-u_{i}^{(t)}=w_{i}^{(t)} \leq 0 \text { on } \Sigma_{t}
$$

(for every $i=1, \ldots, m$ and every $\left.t \in\left(\mathbf{a}_{\Omega}, \tau_{0}\right)\right)$.

We finally claim that, by the Strong Maximum Principle for $C^{1}$-subsolutions, we have

(3.14) $u_{i}<u_{i}^{(t)}$ on $\Sigma_{t}$ for every $i=1, \ldots, m$ and every $t \in\left(\mathbf{a}_{\Omega}, \tau_{0}\right)$.

Indeed, let $i \in\{1, \ldots, m\}$ and $t \in\left(\mathbf{a}_{\Omega}, \tau_{0}\right)$ be arbitrarily fixed. Clearly, the set $\Sigma_{t}$ is (open and) connected. Moreover, since the (vector-valued) map $W_{t}=U-U_{t}$ solves $(3.2)$ and $c_{i j}(\cdot ; t) \geq 0$ for every $j \neq i$, we have

$$
-\Delta w_{i}^{(t)}=\sum_{j=1}^{m} c_{i j}(\cdot ; t) w_{j}^{(t)} \leq c_{i i}(\cdot ; t) w_{i}^{(t)} \quad\left(\text { as } u_{i} \leq u_{i}^{(t)}\right) .
$$

We explicitly point out that the above inequality has to be intended in the weak sense of distributions on $\Sigma_{t}$. This means precisely that

$$
\int_{\Sigma_{t}}\left\langle\nabla w_{i}^{(t)}, \nabla \varphi\right\rangle \mathrm{d} x \leq \int_{\Sigma_{t}} c_{i i}(\cdot ; t) w_{i}^{(t)} \mathrm{d} x,
$$

for all $\varphi \in C_{0}^{\infty}\left(\Sigma_{t}, \mathbb{R}\right)$ with $\varphi \geq 0$ on $\Sigma_{t}$. 
From this, taking into account (3.5) we get

$$
-\Delta w_{i}^{(t)}+\left(\mathbf{c}_{f}-c_{i i}(\cdot ; t)\right) w_{i}^{(t)} \leq \mathbf{c}_{f} w_{i}^{(t)} \leq 0,
$$

and $\mathbf{c}_{f}-c_{i i}(\cdot ; t) \geq 0$ on $\Sigma_{t}$. Gathering together all these facts, we can invoke the Strong Maximum Principle for $C^{1}$-subsolution (see, e.g., [23]), ensuring that

$$
\text { either } w_{i}^{(t)}<0 \text { or } w_{i}^{(t)} \equiv 0 \text { on } \Sigma_{t} .
$$

Since, by (3.2), we know that the function $w_{t}^{(t)}$ is (strictly) negative on the set $\partial \Sigma_{t} \backslash \Pi_{t}$ (notice $t<\tau_{0}<0$ ), we then conclude that (3.14) holds true.

Finally, on account of (3.14) (and taking into account the very definition of $\mathcal{I})$, we see that $\left(\mathbf{a}_{\Omega}, \tau_{0}\right) \subseteq \mathcal{I}$, whence $\mathcal{I} \neq \varnothing$, and that $\lambda_{0}=\sup \mathcal{I} \geq \tau_{0}>\mathbf{a}_{\Omega}$.

Step II: We now turn to prove that $\lambda_{0}=0$. To this end, following [18], we argue by contradiction and we assume that $\lambda_{0} \in\left(\mathbf{a}_{\Omega}, 0\right)$. Since $W_{\lambda_{0}}$ is continuous on $\Sigma_{\lambda_{0}} \backslash R_{\lambda_{0}}(\Gamma)$, from the very definition of $\lambda_{0}$ we deduce that, for every $i \in\{1, \ldots, m\}$,

$$
w_{i}^{\left(\lambda_{0}\right)} \leq 0 \text { on } \Sigma_{\lambda_{0}} \backslash R_{\lambda_{0}}(\Gamma) \text {, that is, } u_{i} \leq u_{\lambda_{0}} \text { on } \Sigma_{\lambda_{0}} \backslash R_{\lambda_{0}}(\Gamma) .
$$

As a consequence, by the Strong Maximum Principle (for $C^{1}$-subsolutions) we get

$$
w_{i}^{\left(\lambda_{0}\right)}<0 \text { on } \Sigma_{\lambda_{0}} \backslash R_{\lambda_{0}}(\Gamma) \text { for every } i \in\{1, \ldots, m\} .
$$

In fact, taking into account that $W_{\lambda_{0}}$ solves (3.2) and arguing exactly as in the last part of the previous step, we have the following family of inequalities (which has to be intended in the weak sense of distributions on $\left.\Sigma_{\lambda_{0}} \backslash R_{\lambda_{0}}(\Gamma)\right)$ :

$$
-\Delta w_{i}^{\left(\lambda_{0}\right)}+\left(\mathbf{c}_{f}-c_{i i}\left(\cdot ; \lambda_{0}\right)\right) w_{i}^{\left(\lambda_{0}\right)} \leq \mathbf{c}_{f} w_{i}^{\left(\lambda_{0}\right)} \leq 0
$$

for every $i \in\{1, \ldots, m\}$. Moreover, since $\mathbf{c}_{f}-c_{i i}\left(\cdot ; \lambda_{0}\right) \geq 0$ on $\Sigma_{\lambda_{0}} \backslash$ $R_{\lambda_{0}}(\Gamma)$ (see (3.5)) and since, by Lemma 2.4(1), the set $\Sigma_{\lambda_{0}} \backslash R_{\lambda_{0}}(\Gamma)$ is open and connected (see also Remark 2.5), we are entitled to apply the Strong Maximum Principle for $C^{1}$-subsolution. Hence,

either $w_{i}^{\left(\lambda_{0}\right)}<0$ or $w_{i}^{\left(\lambda_{0}\right)} \equiv 0$ on $\Sigma_{\lambda_{0}} \backslash R_{\lambda_{0}}(\Gamma) \quad$ (for any $i \in\{1, \ldots, m\}$ ). 
Finally, since we know that the functions $w_{1}^{\left(\lambda_{0}\right)}, \ldots, w_{m}^{\left(\lambda_{0}\right)}$ are (strictly) negative on the set $\left(\partial \Sigma_{\lambda_{0}} \backslash \Pi_{\lambda_{0}}\right) \backslash R_{\lambda_{0}}(\Gamma)$ (as $\lambda_{0}<0$, see $\left.(3.2)\right)$, we conclude that (3.16) holds true.

Now we have established (3.16), we then turn to prove the following assertion: in correspondence to every compact set $K \subseteq \Sigma_{\lambda_{0}} \backslash R_{\lambda_{0}}(\Gamma)$ with Lipschitz boundary $\partial K$, it is possible to find a small $\epsilon=\epsilon\left(K, \lambda_{0}\right) \epsilon$ $\left(0,\left|\lambda_{0}\right| / 2\right)$ such that

(a) $K \subseteq \Sigma_{\lambda} \backslash R_{\lambda}(\Gamma)$ for every $\lambda \in\left[\lambda_{0}, \lambda_{0}+\epsilon\right]$;

(b) $\left(w_{i}^{(\lambda)}\right)^{+} \equiv 0$ on $K$ for every $i \in\{1, \ldots, m\}$ and every $\lambda \in\left(\lambda_{0}, \lambda_{0}+\epsilon\right]$;

(c) for every $i \in\{1, \ldots, m\}$ and every $\lambda \in\left(\lambda_{0}, \lambda_{0}+\epsilon\right]$ we have

$$
\left\|\nabla\left(w_{i}^{(\lambda)}\right)^{+}\right\|_{L^{2}\left(\Sigma_{\lambda} \backslash K\right)} \leq \mathbf{c}_{f} \theta_{n}^{2}\left(\Sigma_{\lambda} \backslash K\right) \sum_{j=1}^{m}\left\|\nabla\left(w_{j}^{(\lambda)}\right)^{+}\right\|_{L^{2}\left(\Sigma_{\lambda} \backslash K\right)}
$$

where $\theta_{n}\left(\Sigma_{\lambda} \backslash K\right)=\Theta\left|\Sigma_{\lambda} \backslash K\right|^{1 / n}$ (see Lemma 2.10).

We explicitly observe that, if $\epsilon<\left|\lambda_{0}\right| / 2$, we have

$$
\left[\lambda_{0}, \lambda_{0}+\epsilon\right] \subseteq\left(\mathbf{a}_{\Omega}, 0\right) .
$$

Let now $K \subseteq \Sigma_{\lambda_{0}} \backslash R_{\lambda_{0}}(\Gamma)$ be an arbitrarily fixed compact set. Since both $K$ and $R_{\lambda_{0}}(\Gamma)$ are closed, it is very easy to recognize that there exists a suitable $\nu=\nu\left(K, \lambda_{0}\right)>0$, which we can assume to be smaller than $\left|\lambda_{0}\right| / 2$, such that

$$
K \subseteq \Sigma_{\lambda} \backslash R_{\lambda}(\Gamma) \text { for every } \lambda \in\left[\lambda_{0}, \lambda_{0}+\nu\right] .
$$

Moreover, on account of (3.16) (and remembering that $W_{\lambda_{0}}$ is continuous on $\left.\Sigma_{\lambda_{0}} \backslash R_{\lambda_{0}}(\Gamma)\right)$, it is possible to find a real constant $M_{0}<0$ such that

$$
w_{i}^{\left(\lambda_{0}\right)} \leq M_{0}<0 \text { on } \Sigma_{\lambda_{0}} \backslash R_{\lambda_{0}}(\Gamma) \text { for every } i \in\{1, \ldots, m\} .
$$

Since, for every fixed $i \in\{1, \ldots, m\}$ and every $\lambda \in\left[\lambda_{0}, \lambda_{0}+\nu\right]$, the function

$$
(x, \lambda) \mapsto w_{i}^{(\lambda)}(x)=u_{i}(x)-u_{i}^{(\lambda)}(x)
$$

is (well-defined and) uniformly continuous on $K \times\left[\lambda_{0}, \lambda_{0}+\nu\right]$ (as it follows from (3.19)), there exists a real $\epsilon=\epsilon\left(K, \lambda_{0}\right) \in(0, \nu)$ (hence, $\left.\epsilon<\left|\lambda_{0}\right| / 2\right)$ such that

$$
w_{i}^{(\lambda)}(x)<w_{i}^{\left(\lambda_{0}\right)}(x)+\frac{\left|M_{0}\right|}{2} \stackrel{(3.20)}{\leq} \frac{M_{0}}{2}<0,
$$

for all $x \in K$ and for all $\lambda \in\left[\lambda_{0}, \lambda_{0}+\epsilon\right]$. Summing up, if $\lambda \in\left[\lambda_{0}, \lambda_{0}+\epsilon\right]$, we have $K \subseteq \Sigma_{\lambda} \backslash R_{\lambda}(\Gamma)$ and $\left(w_{i}^{(\lambda)}\right)^{+} \equiv 0$ on $K$. 
We then turn to prove (3.18). To this end, let $i \in\{1, \ldots, m\}$ and let $\lambda \in\left(\lambda_{0}, \lambda_{0}+\epsilon\right]$ be arbitrarily fixed. We consider the (double) sequence of functions defined by

$$
\varphi_{h, k}:= \begin{cases}\left(w_{i}^{(\lambda)}\right)^{+} \phi_{h}^{2} \psi_{k}^{2} & \text { on } \in \Sigma_{\lambda}, \\ 0 & \text { on } \mathbb{R}^{n} \backslash \Sigma_{\lambda},\end{cases}
$$

where $\left\{\phi_{h}\right\}_{h \in \mathbb{N}}$ is the sequence defined in (2.22) and associated with $\gamma_{\lambda}=\partial \Omega \cap \Pi_{\lambda}$, whilst $\left\{\psi_{k}\right\}_{k \in \mathbb{N}}$ is the sequence defined in (2.19) and associated with $R_{\lambda}(\Gamma)$. Actually, the functions $\varphi_{h, k}$ also depend on the fixed $i$ and $\lambda$. However, in order to avoid cumbersome notations, we prefer to not keep trace of this dependence in the sequel).

By Lemma 2.8, for every $h, k \in \mathbb{N}$ we have $\varphi_{h, k} \in \operatorname{Lip}\left(\overline{\Sigma_{\lambda}}\right)$ and $\varphi_{h, k} \equiv$ 0 on $\partial \Sigma_{\lambda}$. Moreover, by (3.21), there exists an open neighborhood $\mathcal{U} \subseteq$ $\Sigma_{\lambda} \backslash R_{\lambda}(\Gamma)$ of $K$ such that

$$
\left(w_{i}^{(\lambda)}\right)^{+} \equiv 0 \text { on } \mathcal{U}, \quad \text { whence } \varphi_{h, k} \equiv 0 \text { on } \mathcal{U} \text { for every } h, k \in \mathbb{N} .
$$

Gathering together all these facts, we deduce that

$$
\varphi_{h, k} \in H_{0}^{1}\left(\Sigma_{\lambda} \backslash K\right) \text {. }
$$

Furthermore, since $\varphi_{h, k} \rightarrow\left(w_{i}^{(\lambda)}\right)^{+}$in $H_{0}^{1}\left(\Sigma_{\lambda}\right)$ as $h, k \rightarrow \infty$ (see Lemma 2.9), we also get

$$
\left(w_{i}^{(\lambda)}\right)^{+} \in H_{0}^{1}\left(\Sigma_{\lambda} \backslash K\right) .
$$

By (3.23), and by a standard density argument, we are entitled to use the function $\varphi_{h, k}$ (for every fixed $h, k \in \mathbb{N}$ ) as test function in (3.4), obtaining (see also (2.26))

$$
\begin{aligned}
\int_{\Sigma_{\lambda}}\left|\nabla\left(w_{i}^{(\lambda)}\right)^{+}\right|^{2} \phi_{h}^{2} \psi_{k}^{2} \mathrm{~d} x+2 \int_{\Sigma_{\lambda}}\left(w_{i}^{(\lambda)}\right)^{+} \psi_{k}^{2} \phi_{h}\left\langle\nabla w_{i}^{(\lambda)}, \nabla \phi_{h}\right\rangle \mathrm{d} x \\
\quad+2 \int_{\Sigma_{\lambda}}\left(w_{i}^{(\lambda)}\right)^{+} \phi_{h}^{2} \psi_{k}\left\langle\nabla w_{i}^{(\lambda)}, \nabla \psi_{k}\right\rangle \mathrm{d} x \\
=\int_{\Sigma_{\lambda}}\left\langle\nabla w_{i}^{(\lambda)}, \nabla \varphi_{h, k}\right\rangle \mathrm{d} x=\sum_{j=1}^{m} \int_{\Sigma_{\lambda}} c_{i j}(\cdot ; \lambda) w_{j}^{(\lambda)} \varphi_{h, k} \mathrm{~d} x \\
=\sum_{j=1}^{m} \int_{\Sigma_{\lambda}} c_{i j}(\cdot ; \lambda)\left(w_{i}^{(\lambda)}\right)^{+} w_{j}^{(\lambda)} \phi_{h}^{2} \psi_{k}^{2} \mathrm{~d} x .
\end{aligned}
$$


From this, by $(3.22),(3.5)$, and the fact that $c_{i j}(\cdot ; \lambda) \geq 0$ if $j \neq i$, we get

$$
\begin{aligned}
\int_{\Sigma_{\lambda} \backslash K}\left|\nabla\left(w_{i}^{(\lambda)}\right)^{+}\right|^{2} \phi_{h}^{2} \psi_{k}^{2} \mathrm{~d} x= & \int_{\Sigma_{\lambda}}\left|\nabla\left(w_{i}^{(\lambda)}\right)^{+}\right|^{2} \phi_{h}^{2} \psi_{k}^{2} \mathrm{~d} x \\
\leq & 2 \int_{\Sigma_{\lambda} \backslash K}\left(w_{i}^{(\lambda)}\right)^{+} \psi_{k}^{2} \phi_{h}\left|\nabla w_{i}^{(\lambda)}\right|\left|\nabla \phi_{h}\right| \mathrm{d} x \\
& +2 \int_{\Sigma_{\lambda} \backslash K}\left(w_{i}^{(\lambda)}\right)^{+} \phi_{h}^{2} \psi_{k}\left|\nabla w_{i}^{(\lambda)}\right|\left|\nabla \psi_{k}\right| \mathrm{d} x \\
& +\mathbf{c}_{f} \sum_{j=1}^{m} \int_{\Sigma_{\lambda} \backslash K}\left(w_{i}^{(\lambda)}\right)^{+}\left(w_{j}^{(\lambda)}\right)^{+} \phi_{h}^{2} \psi_{k}^{2} \mathrm{~d} x .
\end{aligned}
$$

We now observe that, since $\nabla w_{i}^{(\lambda)}=\nabla\left(w_{i}^{(\lambda)}\right)^{+}$almost everywhere on the set $\left\{w_{i}^{(\lambda)}>0\right\}$, the above inequality can be re-written as follows:

$$
\begin{aligned}
\int_{\Sigma_{\lambda} \backslash K} & \left|\nabla\left(w_{i}^{(\lambda)}\right)^{+}\right|^{2} \phi_{h}^{2} \psi_{k}^{2} \mathrm{~d} x \\
\leq & \int_{\Sigma_{\lambda} \backslash K} 2\left(\left(w_{i}^{(\lambda)}\right)^{+} \psi_{k}\left|\nabla \phi_{h}\right|\right)\left(\psi_{k} \phi_{h}\left|\nabla\left(w_{i}^{(\lambda)}\right)^{+}\right|\right) \mathrm{d} x \\
& +\int_{\Sigma_{\lambda} \backslash K} 2\left(\left(w_{i}^{(\lambda)}\right)^{+} \phi_{h}\left|\nabla \psi_{k}\right|\right)\left(\phi_{h} \psi_{k}\left|\nabla\left(w_{i}^{(\lambda)}\right)^{+}\right|\right) \mathrm{d} x \\
& +\mathbf{c}_{f} \sum_{j=1}^{m} \int_{\Sigma_{\lambda} \backslash K}\left(w_{i}^{(\lambda)}\right)^{+}\left(w_{j}^{(\lambda)}\right)^{+} \phi_{h}^{2} \psi_{k}^{2} \mathrm{~d} x .
\end{aligned}
$$

From this, by using the classical Young's inequality

$$
2 a b \leq 4 a^{2}+\frac{1}{4} b^{2} \quad \text { (holding true for every } a, b \geq 0 \text { ) }
$$

on the integrands of the first two integrals in the right-hand side of (3.25), we get

$$
\begin{aligned}
\frac{1}{2} \int_{\Sigma_{\lambda} \backslash K}\left|\nabla\left(w_{i}^{(\lambda)}\right)^{+}\right|^{2} \phi_{h}^{2} \psi_{k}^{2} \mathrm{~d} x \leq & 4 \int_{\Sigma_{\lambda} \backslash K}\left[\left(w_{i}^{(\lambda)}\right)^{+}\right]^{2} \psi_{k}^{2}\left|\nabla \phi_{h}\right|^{2} \mathrm{~d} x \\
& +4 \int_{\Sigma_{\lambda} \backslash K}+\left[\left(w_{i}^{(\lambda)}\right)^{+}\right]^{2} \phi_{h}^{2}\left|\nabla \psi_{k}\right|^{2} \mathrm{~d} x \\
& +\mathbf{c}_{f} \sum_{j=1}^{m} \int_{\Sigma_{\lambda} \backslash K}\left(w_{i}^{(\lambda)}\right)^{+}\left(w_{j}^{(\lambda)}\right)^{+} \phi_{h}^{2} \psi_{k}^{2} \mathrm{~d} x .
\end{aligned}
$$


To proceed further towards the proof of (3.18) we observe that, since $u_{1}, \ldots, u_{m}$ are positive on $\Omega \backslash \Gamma$ and $R_{\lambda}\left(\Sigma_{\lambda}\right) \subseteq \Omega$ (by convexity), we have

$$
0 \leq\left(w_{i}^{(\lambda)}\right)^{+}=\left(u_{i}-u_{i}^{(\lambda)}\right)^{+} \leq u_{i}, \quad \text { on } \Sigma_{\lambda} \backslash R_{\lambda}(\Gamma)
$$

As a consequence, since $u_{i}$ is continuous on the set $\overline{\Sigma_{\lambda}} \subseteq \bar{\Omega} \backslash \Gamma$ (recall that, by assumption $\lambda \leq \lambda_{0}+\epsilon<0$ and $\left.\Gamma \subseteq\left\{x_{1}=0\right\}\right)$, we get

$$
\begin{gathered}
\frac{1}{2} \int_{\Sigma_{\lambda} \backslash K}\left|\nabla\left(w_{i}^{(\lambda)}\right)^{+}\right|^{2} \phi_{h}^{2} \psi_{k}^{2} \mathrm{~d} x \leq 4\|u\|_{L^{\infty}\left(\Sigma_{\lambda_{0}+\epsilon}\right)}^{2} \int_{\Sigma_{\lambda} \backslash K} \psi_{k}^{2}\left|\nabla \phi_{h}\right|^{2} \mathrm{~d} x \\
+4\|u\|_{L^{\infty}\left(\Sigma_{\lambda_{0}+\epsilon}\right)}^{2} \int_{\Sigma_{\lambda} \backslash K} \phi_{h}^{2}\left|\nabla \psi_{k}\right|^{2} \mathrm{~d} x \\
\quad+\mathbf{c}_{f} \sum_{j=1}^{m} \int_{\Sigma_{\lambda} \backslash K}\left(w_{i}^{(\lambda)}\right)^{+}\left(w_{j}^{(\lambda)}\right)^{+} \phi_{h}^{2} \psi_{k}^{2} \mathrm{~d} x
\end{gathered}
$$

(by $(2.20),(2.21),(2.23)$, and $(2.24))$

$$
\leq 16\|u\|_{L^{\infty}\left(\Sigma_{\lambda_{0}+\epsilon}\right)}^{2}\left(\frac{1}{h}+\frac{1}{k}\right)+\mathbf{c}_{f} \sum_{j=1}^{m} \int_{\Sigma_{\lambda} \backslash K}\left(w_{i}^{(\lambda)}\right)^{+}\left(w_{j}^{(\lambda)}\right)^{+} \mathrm{d} x .
$$

Letting $h, k \rightarrow \infty$ (and using Fatou's Lemma, see (2.20) and (2.23)), we then obtain

$$
\begin{array}{r}
\frac{1}{2} \int_{\Sigma_{\lambda} \backslash K}\left|\nabla\left(w_{i}^{(\lambda)}\right)^{+}\right|^{2} \mathrm{~d} x \leq \mathbf{c}_{f} \sum_{j=1}^{m} \int_{\Sigma_{\lambda} \backslash K}\left(w_{i}^{(\lambda)}\right)^{+}\left(w_{j}^{(\lambda)}\right)^{+} \mathrm{d} x \\
\leq \mathbf{c}_{f}\left\|\left(w_{i}^{(\lambda)}\right)^{+}\right\|_{L^{2}\left(\Sigma_{\lambda} \backslash K\right)} \sum_{j=1}^{m}\left\|\left(w_{j}^{(\lambda)}\right)^{+}\right\|_{L^{2}\left(\Sigma_{\lambda} \backslash K\right)}
\end{array}
$$

Now, by exploiting (3.24), we can apply (2.30) (for the Sobolev space $\left.H_{0}^{1}\left(\Sigma_{\lambda} \backslash K\right)\right)$ on the right-hand side of (3.26):

$$
\begin{aligned}
& \frac{1}{2}\left\|\nabla\left(w_{i}^{(\lambda)}\right)^{+}\right\|_{L^{2}\left(\Sigma_{\lambda} \backslash K\right)}^{2}=\int_{\Sigma_{\lambda} \backslash K}\left|\nabla\left(w_{i}^{(\lambda)}\right)^{+}\right|^{2} \mathrm{~d} x \\
& \quad \leq \theta_{n}^{2}\left(\Sigma_{\lambda} \backslash K\right) \mathbf{c}_{f}\left\|\nabla\left(w_{i}^{(\lambda)}\right)^{+}\right\|_{L^{2}\left(\Sigma_{\lambda} \backslash K\right)} \sum_{j=1}^{m}\left\|\nabla\left(w_{j}^{(\lambda)}\right)^{+}\right\|_{L^{2}\left(\Sigma_{\lambda} \backslash K\right)} .
\end{aligned}
$$


Finally, to complete the proof of assertion (c) we observe that, if

$$
\left\|\nabla\left(w_{i}^{(\lambda)}\right)^{+}\right\|_{L^{2}\left(\Sigma_{\lambda} \backslash K\right)}=0,
$$

then (3.18) is trivially satisfied. If, instead, (3.28) does not hold, by (3.27) one has

$$
\frac{1}{2}\left\|\nabla\left(w_{i}^{(\lambda)}\right)^{+}\right\|_{L^{2}\left(\Sigma_{\lambda} \backslash K\right)} \leq \theta_{n}^{2}\left(\Sigma_{\lambda} \backslash K\right) \mathbf{c}_{f} \sum_{j=1}^{m}\left\|\nabla\left(w_{j}^{(\lambda)}\right)^{+}\right\|_{L^{2}\left(\Sigma_{\lambda} \backslash K\right)},
$$

and this is precisely the desired (3.18).

Now that we have proved (3.18), we are ready to complete the proof of the present step. To begin with, let $\delta_{0}>0$ be a fixed real number such that

$K_{\delta}:=\left\{x \in \Sigma_{\lambda_{0}} \backslash R_{\lambda_{0}}(\Gamma): \operatorname{dist}\left(x, \partial\left(\Sigma_{\lambda_{0}} \backslash R_{\lambda_{0}}(\Gamma)\right) \geq \delta\right\} \neq \varnothing\right.$, for all $\delta \in\left(0, \delta_{0}\right]$.

Moreover, given any $\delta \in\left(0, \delta_{0}\right]$, let $\epsilon_{\delta}=\epsilon\left(K_{\delta}, \lambda_{0}\right) \in\left(0,\left|\lambda_{0}\right| / 2\right)$ be such that assertions (a)-(c) hold true for every $\lambda \in\left[\lambda_{0}, \lambda_{0}+\epsilon_{\delta}\right]$ (note that $K_{\delta}$ has Lipschitz boundary).

Since $\left|R_{\lambda}(\Gamma)\right|=0$ for every $\lambda \in \mathbb{R}$ (both in the case $n=2$ and in the case $n \geq 3$, see assumption (H.2) and, e.g, [20, Section 4.7]), it is very easy to recognize that

(3.29) for every $\eta>0$ there exists $\delta_{\eta} \in\left(0, \delta_{0}\right)$ such that

$$
\theta_{n}\left(\Sigma_{\lambda} \backslash K_{\delta}\right)<\eta \text { for every } 0<\delta<\delta_{\eta} \text { and every } \lambda \in\left[\lambda_{0}, \lambda_{0}+\epsilon_{\delta}\right] .
$$

Starting from (3.18) and performing an induction argument analogous to that in Step I (in which the information $\theta_{n}\left(\Sigma_{t}\right) \rightarrow 0$ as $t \rightarrow \mathbf{a}_{\Omega}$ is replaced by $(3.29))$, we infer the existence of a small $\sigma \in\left(0, \delta_{0}\right)$ and of a real $C_{m}=C_{m}\left(\mathbf{c}_{f}\right)>0$ such that

$$
\left\|\nabla\left(w_{i}^{(\lambda)}\right)^{+}\right\|_{L^{2}\left(\Sigma_{\lambda} \backslash K_{\sigma}\right)} \leq C_{m} \theta_{n}^{2}\left(\Sigma_{\lambda} \backslash K_{\sigma}\right) \sum_{j \geq i+1}\left\|\nabla\left(w_{j}^{(\lambda)}\right)^{+}\right\|_{L^{2}\left(\Sigma_{\lambda} \backslash K_{\sigma}\right)}
$$

for every $i=1, \ldots, m-1$ and every $\lambda \in\left[\lambda_{0}, \lambda_{0}+\epsilon_{\sigma}\right]$. From this, again arguing exactly as in Step I, we can use a backward induction argument to prove that

$$
\left\|\nabla\left(w_{j}^{(\lambda)}\right)^{+}\right\|_{L^{2}\left(\Sigma_{\lambda} \backslash K_{\sigma}\right)} \leq \mathcal{C}_{m} \theta_{n}^{2}\left(\Sigma_{\lambda} \backslash K_{\sigma}\right)\left\|\nabla\left(w_{m}^{(\lambda)}\right)^{+}\right\|_{L^{2}\left(\Sigma_{\lambda} \backslash K_{\sigma}\right)},
$$

for all $j \in\{1, \ldots, m-1\}$ and every $\lambda \in\left[\lambda_{0}, \lambda_{0}+\epsilon_{\sigma}\right]$. (Here, as usual, $\mathcal{C}_{m}>0$ is a real constant only depending on $\mathbf{c}_{f}$.) By combining (3.30) with (3.31), and by possibly shrinking $\sigma$ if necessary, we obtain (see 
also (3.13) in the last part of Step I and recall that the vector-valued map $W_{\lambda}$ is continuous outside of $R_{\lambda}(\Gamma)$, see (3.1))

$$
w_{i}^{(\lambda)} \leq 0 \text { on } \Sigma_{\lambda} \backslash\left(K_{\sigma} \cup R_{\lambda}(\Gamma)\right),
$$

for all $i \in\{1, \ldots, m\}$ and for all $\lambda \in\left[\lambda_{0}, \lambda_{0}+\epsilon_{\sigma}\right]$. Gathering together (3.32) and assertion (b), we then conclude that

$w_{i}^{(\lambda)} \leq 0$ on $\Sigma_{\lambda} \backslash R_{\lambda}(\Gamma)$ for every $i \in\{1, \ldots, m\}$ and every $\lambda \in\left[\lambda_{0}, \lambda_{0}+\epsilon_{\sigma}\right]$. From this, a last application of the Strong Maximum Principle gives $\left(\right.$ as $\left.\lambda_{0}<0\right)$

$$
u_{i}-u_{i}^{(\lambda)}=w_{i}^{(\lambda)}<0 \text { on } \Sigma_{\lambda} \backslash R_{\lambda}(\Gamma),
$$

for all $i \in\{1, \ldots, m\}$ and for all $\lambda \in\left[\lambda_{0}, \lambda_{0}+\epsilon_{\sigma}\right]$, but this is contradiction with the definition of $\lambda_{0}$. Hence, $\lambda_{0}=0$.

Step III: In this step we prove that all the functions $u_{1}, \ldots, u_{m}$ are symmetric with respect to the hyperplane $\Pi=\left\{x_{1}=0\right\}$. To this end we first observe that, since we know from Step II that $\lambda_{0}=\sup \mathcal{I}=0$ and since $W_{\lambda}$ is continuous outside of $R_{\lambda}(\Gamma)$, one has

$$
u_{i}\left(x_{1}, x_{2}, \ldots, x_{n}\right) \leq u_{i}\left(-x_{1}, x_{2}, \ldots, x_{n}\right)=u_{i}^{(0)}\left(x_{1}, \ldots, x_{n}\right),
$$

for every $i \in\{1, \ldots, m\}$ and every $x \in \Omega_{0}=\Omega \cap\left\{x_{1}<0\right\}$. By applying this result to the vector-valued function $\hat{U}: \Omega \rightarrow \mathbb{R}^{m}$ defined by

$$
\hat{U}(x):=U\left(-x_{1}, x_{2}, \ldots, x_{n}\right)
$$

(which has the same regularity of $U$ and is a solution (1.1)), we obtain

$$
\begin{aligned}
u_{i}\left(-x_{1}, \ldots, x_{n}\right)=\hat{u}_{i} & \left(x_{1}, x_{2}, \ldots, x_{n}\right) \\
& \leq \hat{u}_{i}\left(-x_{1}, x_{2}, \ldots, x_{n}\right)=u_{i}\left(x_{1}, \ldots, x_{n}\right),
\end{aligned}
$$

for every $i \in\{1, \ldots, m\}$ and every $x \in \Omega_{0}$. By combining (3.33) with (3.34) we get

$$
u_{i}\left(-x_{1}, \ldots, x_{n}\right)=u_{i}\left(x_{1}, \ldots, x_{n}\right),
$$

for all $i \in\{1, \ldots, m\}$ and for all $x \in \Omega \cap\left\{x_{1}<0\right\}$, and this proves that $u_{1}, \ldots, u_{m}$ are symmetric with respect to $\Pi$.

Step IV: In this last step we prove (1.3), which clearly implies the monotonicity of the functions $u_{1}, \ldots, u_{n}$ in the $x_{1}$-direction on $\Omega \cap\left\{x_{1}<0\right\}$. To this end we first observe that, again from the fact that $\lambda_{0}=\mathcal{I}=0$ (see Step II), we have

$$
w_{i}^{(\lambda)}=u_{i}-u_{i}^{(\lambda)}<0 \text { on } \Sigma_{\lambda} \backslash R_{\lambda}(\Gamma),
$$


for all $i \in\{1, \ldots, m\}$ and for all $\lambda \in\left(\mathbf{a}_{\Omega}, 0\right)$. Moreover, $w_{i}^{(\lambda)} \equiv 0$ on the hyperplane $\Pi_{\lambda}=\left\{x_{1}=\lambda\right\}$ and, by $(3.17)$,

$$
-\Delta w_{i}^{(\lambda)}+\left(\mathbf{c}_{f}-c_{i i}(\cdot ; \lambda)\right) w_{i}^{(\lambda)} \leq 0 \quad \text { on } \Sigma_{\lambda} \backslash R_{\lambda}(\Gamma)
$$

(where $\mathbf{c}_{f}$ is as in assumption (H.3) and the $c_{i j}(\cdot ; \lambda)$ 's are defined in (3.3)). Since, by the choice of $\mathbf{c}_{f}$, we have $\mathbf{c}_{f}-c_{i i}(\cdot ; \lambda) \geq 0$ on $\Sigma_{\lambda} \backslash R_{\lambda}(\Gamma)$, we are entitled to apply the Hopf's Lemma for $C^{1}$-subsolutions in $[\mathbf{2 7}]$ (see (3.1) and note that $\Sigma_{\lambda} \backslash R_{\lambda}(\Gamma)$ certainly satisfies the interior ball condition at any point of $\left.\Pi_{\lambda} \cap \Omega\right)$ :

$0<\frac{\partial w_{i}^{(\lambda)}}{\partial x_{1}}(x)=2 \frac{\partial u_{i}}{\partial x_{1}}(x), \quad$ for all $i \in\{1, \ldots, m\}$ and every $x \in \Pi_{\lambda} \cap \Omega$,

which clearly implies the desired (1.3). Hence, the proof of Theorem 1.1 is complete.

Acknowledgements. We thank the anonymous referee for the suggestions that improved the presentation of the paper.

\section{References}

[1] A. D. Aleksandrov, Uniqueness theorems for surfaces in the large. I, Amer. Math. Soc. Transl. (2) 21 (1962), 341-354. DOI : 10.1090/trans2/021/09.

[2] A. D. Alexandrov, A characteristic property of spheres, Ann. Mat. Pura Appl. (4) 58 (1962), 303-315. DOI : 10.1007/BF02413056.

[3] E. Berchio, F. Gazzola, and T. Weth, Radial symmetry of positive solutions to nonlinear polyharmonic Dirichlet problems, J. Reine Angew. Math. 2008(620) (2008), 165-183. DOI: 10.1515/CRELLE. 2008.052.

[4] H. Berestycki and L. Nirenberg, On the method of moving planes and the sliding method, Bol. Soc. Brasil. Mat. (N.S.) 22(1) (1991), 1-37. DOI : 10.1007/ BF01244896

[5] S. Biagi, E. Valdinoci, And E. Vecchi, A symmetry result for elliptic systems in punctured domains, Commun. Pure Appl. Anal. 18(5) (2019), 2819-2833. DOI : $10.3934 /$ cpaa. 2019126.

[6] L. Caffarelli, Y. Y. Li, and L. Nirenberg, Some remarks on singular solutions of nonlinear elliptic equations. II: symmetry and monotonicity via moving planes, in: "Advances in Geometric Analysis", Adv. Lect. Math. (ALM) 21, Int. Press, Somerville, MA, 2012, pp. 97-105.

[7] A. Canino and M. Degiovanni, A variational approach to a class of singular semilinear elliptic equations, J. Convex Anal. 11(1) (2004), 147-162.

[8] A. Canino, M. Grandinetti, and B. Sciunzi, Symmetry of solutions of some semilinear elliptic equations with singular nonlinearities, J. Differential Equations 255(12) (2013), 4437-4447. DOI : 10.1016/j.jde.2013.08.014.

[9] A. Canino, L. Montoro, and B. Sciunzi, The moving plane method for singular semilinear elliptic problems, Nonlinear Anal. 156 (2017), 61-69. DOI : 10. 1016/j.na.2017.02.009. 
[10] A. Canino And B. Sciunzi, A uniqueness result for some singular semilinear elliptic equations, Commun. Contemp. Math. 18(6) (2016), 1550084, 9 pp. DOI : $10.1142 / \mathrm{S} 0219199715500844$.

[11] C.-C. Chen And C.-S. Lin, Local behavior of singular positive solutions of semilinear elliptic equations with Sobolev exponent, Duke Math. J. 78(2) (1995), 315-334. DOI : 10.1215/S0012-7094-95-07814-4.

[12] F. Colasuonno and E. Vecchi, Symmetry in the composite plate problem, Commun. Contemp. Math. 21(2) (2019), 1850019, 34 pp. DOI: 10.1142/ S0219199718500190.

[13] F. Colasuonno and E. VecChi, Symmetry and rigidity for the hinged composite plate problem, J. Differential Equations 266(8) (2019), 4901-4924. DOI : 10. $1016 / j \cdot j$ de.2018.10.011.

[14] M. G. Crandall, P. H. Rabinowitz, and L. Tartar, On a Dirichlet problem with a singular nonlinearity, Comm. Partial Differential Equations 2(2) (1977), 193-222. DOI : $10.1080 / 03605307708820029$.

[15] L. Damascelli and F. PaCella, Symmetry results for cooperative elliptic systems via linearization, SIAM J. Math. Anal. 45(3) (2013), 1003-1026. DOI : $10.1137 / 110853534$.

[16] D. G. De Figueiredo, Monotonicity and symmetry of solutions of elliptic systems in general domains, NoDEA Nonlinear Differential Equations Appl. 1(2) (1994), 119-123. DOI : 10.1007/BF01193947.

[17] F. Esposito, Symmetry and monotonicity properties of singular solutions to some cooperative semilinear elliptic systems involving critical nonlinearities, Discrete Contin. Dyn. Syst. 40(1) (2020), 549-577. DOI : 10.3934/dcds. 2020022.

[18] F. Esposito, A. Farina, and B. Sciunzi, Qualitative properties of singular solutions to semilinear elliptic problems, J. Differential Equations 265(5) (2018), 1962-1983. DOI : 10.1016/j.jde.2018.04.030.

[19] F. Esposito, L. Montoro, and B. Sciunzi, Monotonicity and symmetry of singular solutions to quasilinear problems, J. Math. Pures Appl. (9) 126 (2019), 214-231. DOI : 10.1016/j.matpur. 2018.09.005.

[20] L. C. Evans And R. F. Gariepy, "Measure Theory and Fine Properties of Functions", Revised edition, Textbooks in Mathematics, CRC Press, Boca Raton, FL, 2015.

[21] A. Ferrero, F. Gazzola, and T. Weth, Positivity, symmetry and uniqueness for minimizers of second-order Sobolev inequalities, Ann. Mat. Pura Appl. (4) 186(4) (2007), 565-578. DOI : 10.1007/s10231-006-0019-9.

[22] B. Gidas, W. M. Ni, And L. Nirenberg, Symmetry and related properties via the maximum principle, Comm. Math. Phys. 68(3) (1979), 209-243. DOI : 10. $1007 / \mathrm{BF} 01221125$.

[23] D. Gilbarg and N. S. Trudinger, "Elliptic Partial Differential Equations of Second Order", Reprint of the 1998 edition, Classics in Mathematics, SpringerVerlag, Berlin, 2001. DOI : 10.1007/978-3-642-61798-0.

[24] J. Heinonen, T. Kilpeläinen, and O. Martio, "Nonlinear Potential Theory of Degenerate Elliptic Equations", Unabridged republication of the 1993 original, Dover Publications, Inc., Mineola, NY, 2006.

[25] L. Montoro, F. Punzo, And B. Sciunzi, Qualitative properties of singular solutions to nonlocal problems, Ann. Mat. Pura Appl. (4) 197(3) (2018), 941-964. DOI : $10.1007 / \mathrm{s} 10231-017-0710-\mathrm{z}$. 
[26] J. NAsh, Continuity of solutions of parabolic and elliptic equations, Amer. J. Math. 80(4) (1958), 931-954. DOI : 10.2307/2372841.

[27] P. Pucci and J. Serrin, "The Maximum Principle", Progress in Nonlinear Differential Equations and their Applications 73, Birkhäuser Verlag, Basel, 2007. DOI : $10.1007 / 978-3-7643-8145-5$.

[28] B. SCIUnzI, On the moving plane method for singular solutions to semilinear elliptic equations, J. Math. Pures Appl. (9) 108(1) (2017), 111-123. DOI : 10. 1016/j .matpur.2016.10.012.

[29] J. Serrin, A symmetry problem in potential theory, Arch. Rational Mech. Anal. 43 (1971), 304-318. DOI : 10.1007/BF00250468.

[30] B. SiRAKOv, Some estimates and maximum principles for weakly coupled systems of elliptic PDE, Nonlinear Anal. 70(8) (2009), 3039-3046. DOI : 10.1016/j.na. 2008.12.026.

[31] S. Terracini, On positive entire solutions to a class of equations with a singular coefficient and critical exponent, Adv. Differential Equations 1(2) (1996), 241-264.

[32] W. C. Troy, Symmetry properties in systems of semilinear elliptic equations, J. Differential Equations 42(3) (1981), 400-413. DOI : 10.1016/0022-0396(81) 90113-3.

S. Biagi

Dipartimento di Ingegneria Industriale e Scienze Matematiche, Università Politecnica della Marche, Via Brecce Bianche, 60131, Ancona, Italy

E-mail address: s.biagi@dipmat.univpm.it

E. Valdinoci

Department of Mathematics and Statistics, University of Western Australia, 35 Stirling Highway, WA 6009 Crawley, Australia

E-mail address: enrico.valdinoci@uwa.edu.au

E. Vecchi

Dipartimento di Matematica, Università degli Studi di Trento, Via Sommarive 14, 38123, Povo (Trento), Italy

E-mail address: eugenio.vecchi@unitn.it

Primera versió rebuda el 24 d'abril de 2019,

darrera versió rebuda el 2 d'octubre de 2019. 